ARTICLE

\title{
H19 IncRNA alters DNA methylation genome wide by regulating S-adenosylhomocysteine hydrolase
}

Jichun Zhou ${ }^{1,2, \star}$, Lihua Yang ${ }^{1,3, \star}$, Tianyu Zhong ${ }^{1,4, \star}$, Martin Mueller ${ }^{1,5}$, Yi Men $^{1,6}$, Na Zhang ${ }^{7}$, Juanke Xie ${ }^{1,8}$, Karolyn Giang ${ }^{9}$, Hunter Chung ${ }^{9}$, Xueguang Sun ${ }^{9}$, Lingeng Lu ${ }^{10}$, Gordon G. Carmichael ${ }^{7}$, Hugh S. Taylor ${ }^{1} \&$ Yingqun Huang ${ }^{1}$

DNA methylation is essential for mammalian development and physiology. Here we report that the developmentally regulated H19 IncRNA binds to and inhibits S-adenosylhomocysteine hydrolase (SAHH), the only mammalian enzyme capable of hydrolysing S-adenosylhomocysteine (SAH). SAH is a potent feedback inhibitor of S-adenosylmethionine (SAM)-dependent methyltransferases that methylate diverse cellular components, including DNA, RNA, proteins, lipids and neurotransmitters. We show that H19 knockdown activates $\mathrm{SAHH}$, leading to increased DNMT3B-mediated methylation of an IncRNA-encoding gene Nctc1 within the Igf2-H19-Nctc1 locus. Genome-wide methylation profiling reveals methylation changes at numerous gene loci consistent with SAHH modulation by H19. Our results uncover an unanticipated regulatory circuit involving broad epigenetic alterations by a single abundantly expressed IncRNA that may underlie gene methylation dynamics of development and diseases and suggest that this mode of regulation may extend to other cellular components.

\footnotetext{
${ }^{1}$ Department of Obstetrics, Gynecology, and Reproductive Sciences, Yale School of Medicine, New Haven, Connecticut 06510, USA. ${ }^{2}$ Department of Surgical Oncology, Affiliated Sir Run Run Shaw Hospital, Zhejiang University School of Medicine, Hangzhou, Zhejiang 310016, China. ${ }^{3}$ Department of Obstetrics and Gynecology, Tianjin Renmin Hospital, Tianjin 300000, China. ${ }^{4}$ Department of Laboratory Medicine, First Affiliated Hospital of Gannan Medical University, Ganzhou, Jiangxi 341000, China. ${ }^{5}$ Department of Obstetrics and Gynecology, University Hospital, Bern 3012, Switzerland. ${ }^{6}$ Department of Head and Neck Surgery, State Key Laboratory of Oral Diseases, West China Hospital of Stomatology, Sichuan University, Chengdu, Sichuan 610041, China. ${ }^{7}$ Department of Genetics and Genome Sciences, University of Connecticut Health Center, Farmington, Connecticut 06030, USA. ${ }^{8}$ Reproductive Medical Center, Henan Provincial People's Hospital, Zhengzhou, Henan 450003, China. ${ }^{9}$ Zymo Research Corporation, Irvine, California 92614, USA. ${ }^{10}$ Department of Chronic Diseases Epidemiology, Yale School of Public Health, Yale University School of Medicine, New Haven, Connecticut 06520, USA. * These authors contributed equally to this work. Correspondence and requests for materials should be addressed to Y.H. (email: Yingqun.huang@yale.edu).
} 
G ene methylation (5-methyl-cytosine), accomplished by the joint action of three S-adenosylmethionine (SAM)dependent DNA methyltransferases (DNMT1, DNMT3A and DNMT3B), plays a critical role in mammalian development and physiology. Altered gene methylation can cause developmental abnormalities and diseases. Being a dynamic process, methylation at a given gene locus is determined not only by the activity of the DNMTs, which have little sequence specificity, but also by local chromatin structure and modifications (that is, histone methylation and acetylation) that affect their accessibility $^{1-3}$. DNMTs can also be targeted to specific gene loci via direct association with transcription factors ${ }^{4}$. Recent discoveries have marked long noncoding RNAs (lncRNAs) as new important players in DNA methylation regulation. IncRNAs can act in cis (at their site of transcription) or in trans (diffusing to other loci) to recruit chromatin-modifying complexes such as the Polycomb repressive complex 2 (PRC2) to affect chromatin structure and modifications ${ }^{5,6}$. In addition, the noncoding ecCEBPA transcript encoded within the CEBPA gene locus binds to DNMT1 and prevents CEBPA gene methylation ${ }^{7}$. This methylation blockade by DNMT1-interacting RNAs may extend to other genomic loci, although each of these RNAs acts locally, not genome wide ${ }^{7}$.

SAM-dependent methylation is central to the regulation of numerous biological processes. A wide spectrum of cellular components, including DNA, RNA, lipids, proteins and neurotransmitters, is subjected to methylation by SAM-dependent methyltransferases. SAM serves as the methyl-group donor during transmethylation reactions, yielding S-adenosylhomocysteine ( $\mathrm{SAH}$ ) as a by-product, which is a strong feedback inhibitor of most SAM-dependent transmethylation reactions. In mammals, S-adenosylhomocysteine hydrolase (SAHH) is the only known enzyme that catalyses the hydrolysis of SAH to homocysteine and adenosine, thereby relieving the inhibition ${ }^{8}$. A complete loss of SAHH is embryonic lethal ${ }^{9}$, whereas SAHH dysfunction results in numerous pathological consequences such as developmental abnormalities, neurovascular disorders, myopathy, cancer and childhood death ${ }^{10,11}$. Furthermore, inhibition of SAHH elicits antiviral effects ${ }^{12}$. Despite the pivotal roles that SAHH plays in a broad range of biological processes, how its activity is regulated remains poorly understood. Being an extraordinarily conserved enzyme (the human and mouse SAHH proteins share $97 \%$ identity), SAHH functions as a tetramer with cofactor $\mathrm{NAD}^{+} / \mathrm{NADH}$ bound to each subunit. Interaction with adenosine or copper inhibits SAHH activity ${ }^{8}$. Recently, lysine acetylation of SAHH has been reported to alter the structure and activity of this enzyme ${ }^{13}$. However, the biological significance of regulation of SAHH by these molecules and by acetylation is unclear. In addition, the possibility of SAHH regulation by other mechanisms remains to be explored.

The developmentally regulated imprinted $H 19$, together with its co-regulated gene Igf2, plays important roles in embryo development and growth control and has been associated with human genetic disorders ${ }^{14}$. H19 is highly expressed in human and mouse placentas and fetal tissues as well as in a subset of postnatal and adult tissues including skeletal muscle ${ }^{15,16}$, heart ${ }^{17-19}$, haematopoietic stem cells $^{20}$ and endometrium ${ }^{21}$. In addition, aberrant $H 19$ expression has been detected in diverse human malignancies ${ }^{22}$. H19 encodes a polyadenylated IncRNA of $\sim 2.6 \mathrm{~kb}$, which is predominantly cytoplasmic with a minor fraction also found in the nucleus ${ }^{14,23}$. H19 is a multifunctional IncRNA that has activities both in the nucleus and in the cytoplasm. Using genetically modified mouse models and cell culture systems, H19 has been shown to interact with the methylCpG-binding domain protein 1, that recruits repressive histone marks to imprinted network genes to inhibit transcription, thereby contributing to embryo growth regulation ${ }^{23}$. H19 also serves as a microRNA precursor for miR-675 that acts to inhibit placental growth ${ }^{24}$, maintain adult haematopoietic stem cells ${ }^{20}$, stimulate skeletal muscle differentiation and regeneration ${ }^{15}$ and promote oncogenesis ${ }^{25,26}$. The oncogenic property of $H 19$ is also attributed to its full-length processed transcript that targets PRC2 (through binding to EZH2, the histone lysine methyltransferase component of PRC2) to genes that promote cancer metastasis ${ }^{27}$. In addition to these nuclear functions, $\mathrm{H} 19$ directly binds to the RNA-binding protein $\mathrm{K}$ homology-type splicing-regulatory protein in the cytoplasm to control myogenic differentiation ${ }^{28}$. Further, H19 acts as a molecular sponge for microRNA let-7, contributing to the regulation of muscle differentiation ${ }^{29}$, glucose metabolism $^{16}$, tumour metastasis ${ }^{30}$ and endometrium development ${ }^{21}$.

In this report we show that $\mathrm{H} 19$ binds to SAHH and inhibits its function both in vivo and in vitro. This interaction prevents SAHH from hydrolysing SAH that blocks DNA methylation by DNMT3B at numerous genomic loci. This differs from what has been described before for other $\operatorname{lncRNAs}$. We propose that this novel epigenetic regulatory mechanism may underlie the DNA methylation dynamics associated with development and diseases and that this mode of regulation may extend to other cellular components.

\section{Results}

SAHH interacts with $\mathrm{H} 19$ in ribonucleoprotein complexes. In search of possible new functions of H19, we performed RNA affinity chromatography using an S1 aptamer-tagged $H 19$ (pH19-S1; untagged $\mathrm{pH} 19$ or S1 tag only as negative controls) ${ }^{29}$ to identify H19-associated protein components (Fig. 1a). The indicated plasmids were each transfected into HEK293 cells that do not express endogenous H19. Purified ribonucleoproteins (RNPs) were resolved using SDS-PAGE, followed by Coomassie blue staining. A protein band with a molecular size of $\sim 48 \mathrm{kDa}$ that was specific to pH19-S1 (Fig. 1b, left lane marked with a red arrow) was cut out and subjected to protein mass spectrometry analysis. A number of proteins were identified including $\mathrm{SAHH}$, nuclear factor 45, propionyl-CoA carboxylase, methylcrotonoylCoA carboxylase, mitochondrial elongation factor Tu (EF-Tu), creatine kinase B-type, $40 \mathrm{~S}$ ribosomal protein SA, multifunctional protein ADE2 isoform 2 and heterogeneous nuclear RNP D. We decided to focus on SAHH for several reasons. First, SAHH belongs to a large family of proteins that use $\mathrm{NAD}(\mathrm{P})^{+} / \mathrm{NAD}(\mathrm{P}) \mathrm{H}$ as cofactors via the dinucleotide-binding Rossmann-fold (a protein structural motif that binds nucleotides, especially the cofactor $\mathrm{NAD})^{8}$. Second, a dozen enzymes harbouring the Rossmann-fold have been reported to bind polyadenylated RNAs in HeLa cells ${ }^{31}$. Third, the Rossmann-fold is an RNA-binding domain for both glyceraldehyde-3-phosphate dehydrogenase (GAPDH) and lactate dehydrogenase (LDH) and mediates their binding to AU- and U-rich RNA elements ${ }^{32,33}$. Finally, SAHH is the only mammalian enzyme capable of breaking down SAH, a potent product inhibitor of most SAM-dependent transmethylation reactions.

Western blot analysis of affinity-purified RNPs revealed association of SAHH with pH19-S1 but not with pH19 (Fig. 1c, top blot, compare lane 3 with lane 4). The RNA-binding protein HuR, previously shown to bind $H 19$ (ref. 24), was also in complex with pH19-S1 under the same conditions (middle blot, lane 4), while beta-tubulin was not (bottom blot, lane 4). Taken together, these results supported the notion that the SAHH-H19 interaction was specific. Next, we performed RNA immunoprecipitation (RIP) experiments using a monoclonal antibody specific to SAHH with myotubes derived from mouse myoblasts to determine 
a

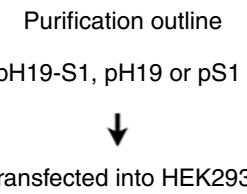

$\downarrow$
Cell lysate preparation
$\downarrow$
Streptavidin affinity purification

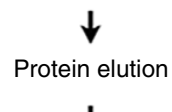

$\downarrow$

SDS-PAGE

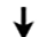

Mass spec/western blot analysis b Commassie staining

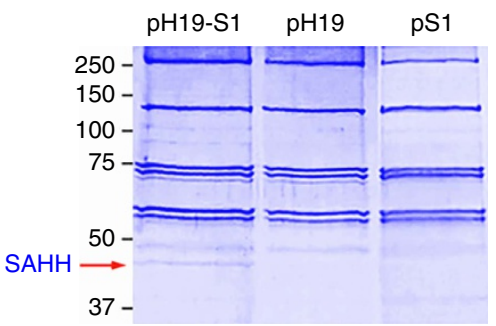

C

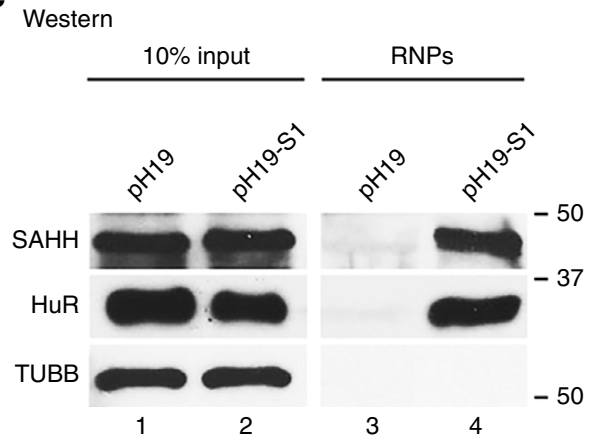

d $\mathrm{RIP}+\mathrm{RT}-\mathrm{qPCR}$

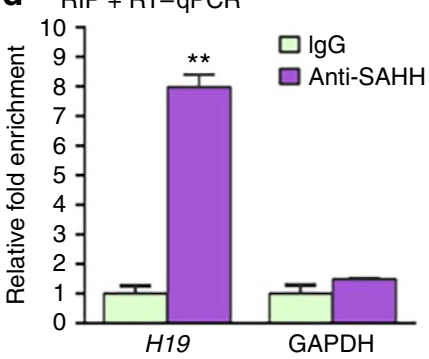

e $R T-q P C R$

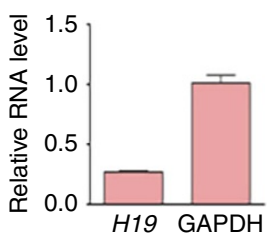

f RIP + Western

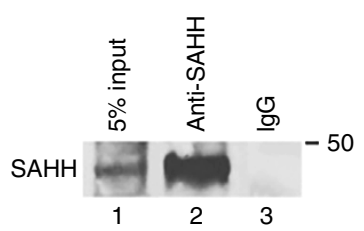

g Igf2-H19-Nctc1 locus

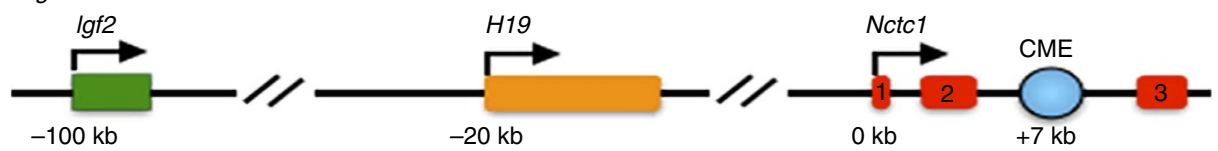

Figure 1 | SAHH interacts with H19 in RNPs. (a) Schematic outline of purification of H19-associated RNPs and protein component identification. (b) Image of a Commassie staining gel with protein size markers (in $\mathrm{kDa}$ ) labelled on the left. The $\mathrm{SAHH}$ protein band is marked with a red arrow. (c) pH19-S1 or pH19 were transfected into HEK293 cells, followed by affinity purification as outlined in a. Purified RNPs were resolved using 10\% SDS-PAGE, followed by western blot analysis using rabbit polyclonal antibodies specific for SAHH (top), HuR (middle) or beta-tubulin (bottom). Ten percent input was loaded on lanes 1 and 2. Molecular marker positions in $\mathrm{kDa}$ are shown on the right. (d) RIP with mouse monoclonal anti-SAHH or pre-immune lgGs from myotube extracts. RNA levels in immunoprecipitates were determined using reverse transcription and qPCR. Levels of H19 and GAPDH mRNA are presented as fold enrichment in anti-SAHH relative to lgG immunoprecipitates. Student's $t$-tests were used to compare differences between quantitative variables. Numbers are mean \pm s.d. $(n=3) .{ }^{\star \star} P<0.01$. (e) Relative RNA levels of $H 19$ and GAPDH in myotubes. (f) Immunoprecipitation using monoclonal anti-SAHH (lane 2) or IgG (lane 3), followed by western blot analysis using a rabbit polyclonal anti-SAHH. Five percent input was loaded in lane 1. The SAHH band is marked. (g) Cartoon depiction of the Igf2-H19-Nctc1 locus (not drawn to scale). The three exons of Nctc1 are marked as red boxes, and the CME as a blue dot. The transcription start site of $\mathrm{Nctc} 1$ is set as $0 \mathrm{~kb}$.

whether SAHH associates with endogenous $H 19$ Both $H 19$ and SAHH were predominantly localized to the cytoplasm of the myotubes (Supplementary Fig. 1). We observed a nearly eightfold enrichment of H19 in SAHH-containing RNPs relative to control IgG immunoprecipitates (Fig. 1d, left column), whereas GAPDH mRNA, expressed at approximately fourfold higher level than that of $H 19$ (Fig. 1e), did not detectably associate with the SAHH-containing RNPs (Fig. 2e, right column). The specificity of the monoclonal SAHH antibody was confirmed using RIP and western blot analysis (Fig. 1f). As expected, we observed preferential enrichment of $\mathrm{H} 19$ in HuR-containing RNPs using a polyclonal $\mathrm{HuR}$ antibody under the same conditions (Supplementary Fig. 2). Collectively, we concluded that SAHH interacts with H19 in RNPs in both human and mouse cells.

H19 knockdown activates SAHH. What could be the functional significance of the SAHH-H19 interaction? We hypothesized that binding to $H 19$ might interfere with SAHH's ability to hydrolyse SAH to homocysteine and adenosine. To test this possibility, we used the well-characterized, imprinted Igf2-H19-Nctcl gene locus in mouse skeletal muscle cells as a model system. The regulation 
a

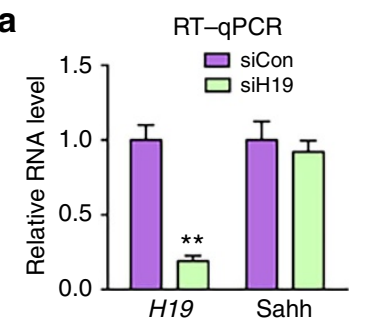

b In vivo SAHH activity

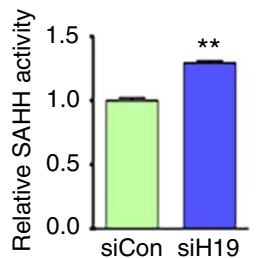

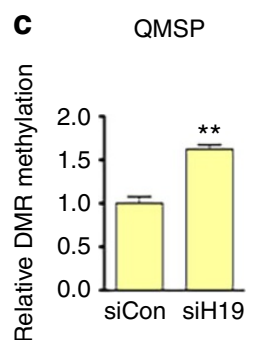

d Sequence of DMR

chr7 142551263

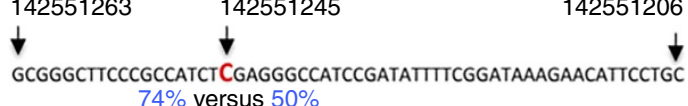

$D M R$ $74 \%$ versus $50 \%$

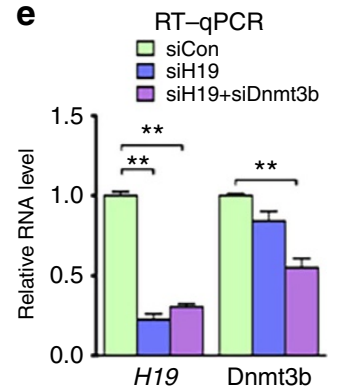

h

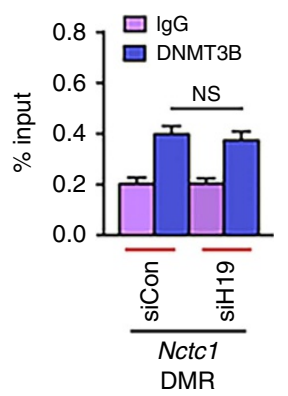

f

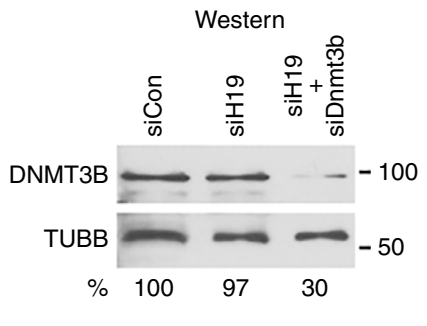

i

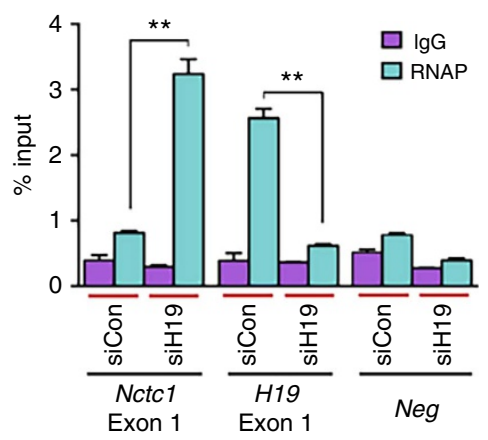

g

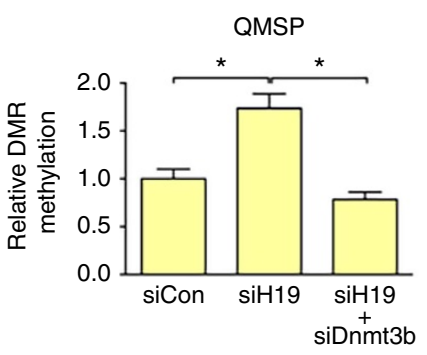

j RT-qPCR

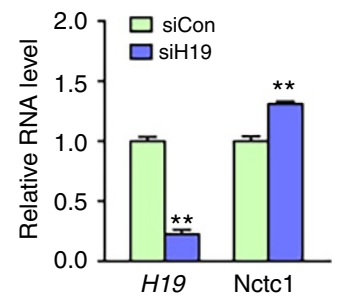

Figure 2 | DNMT3B contributes to SAHH-mediated, H19-dependent methylation change in CME. (a) Myotubes were transfected with siCon or siH19, followed by RNA extraction and RT-qPCR analysis $48 \mathrm{~h}$ later. Relative RNA levels of $H 19$ and Sahh are presented. (b) In vivo SAHH activity assessed $48 \mathrm{~h}$ post-siRNA transfection, as described in a. (c) Relative DMR methylation assessed using QMSP. (d) Sequence of the DMR amplified using the QMSP primers, with the differentially methylated cytosine highlighted in red. Numbers above the sequence mark positions of the indicated nucleotides in the chromosome. Numbers below indicate percentage of methylation in siH19 (left) versus siCon (right) cells, as determined using genome-wide methylation analysis. (e) Myotubes were transfected with siCon, siH19 or siH19 plus siDnmt3b, followed by RNA extraction and RT-qPCR analysis $48 \mathrm{~h}$ post transfection. Relative RNA levels of $\mathrm{H} 19$ and Dnmt3b are presented. (f) Western blot results representative of three independent transfection experiments (performed as described in $\mathbf{e}$ ) are shown. Antibodies specific for DNMT3B and beta-tubulin were used on the top and bottom blots, respectively. Numbers underneath the blots indicate DNMT3B protein levels after normalization against TUBB loading controls with that in siCon cells arbitrarily set as $100 \%$. (g) Myotubes were transfected with siCon, siH19 or siH19 plus siDnmt3b. DMR methylation was assessed 48 host transfection using QMSP.

(h) DNMT3B occupation at CME. Myotubes were transfected with siCon or siH19. ChIP analysis was performed $48 \mathrm{~h}$ later using anti-DNMT3B antibody to evaluate binding of DNMT3B at the DMR. ns, not statistically different. (i) Association of activated RNAP with Nctc1 and H19. Myotubes were transfected as described in $\mathbf{h}$. ChIP analysis was performed using anti-RNAP antibody. (j) Relative RNA levels of H19 and Nctc1 following siRNA transfection. Student's $t$-tests were performed to compare differences between quantitative variables. Numbers are mean \pm s.d. $(n=3) .{ }^{\star} P<0.05,{ }^{\star \star} P<0.01$.

of expression of these genes requires a shared enhancer that is located $20 \mathrm{~kb}$ downstream of $\mathrm{H} 19$ (or $100 \mathrm{~kb}$ downstream of $\operatorname{Igf} 2)^{34}$. This enhancer functions in an unique manner in that it needs both a short core element of $\sim 300$ bp (called core muscle enhancer, or CME) and transcription of the lncRNA-encoding gene $N c t c 1$ within the same enhancer region ${ }^{35}$ (Fig. 1g). The CME resides within the gene body of Nctcl in the second intron and is
$7 \mathrm{~kb}$ downstream of the $N c t c 1$ promoter. In adult muscle cells where Nctc1 has lost its imprinting ${ }^{36}$, activation of the Nctc1 promoter by CME has a negative impact on H19 expression. Promoter competition (the Nctc1 promoter recruits RNA polymerase II (RNAP) for its own transcription at the expense of activation of the H19 promoter) has been proposed as a possible underlying mechanism ${ }^{36}$. Indeed, in myotubes 
differentiated in vitro from mouse myoblasts $N c t c 1$ expression was inversely correlated with that of H19. Consistent with the promoter competition model, association of RNAP with Nctc1 was also inversely correlated with H19 transcriptional activation $^{36}$. Intriguingly, the CME contains a 219-bp CpG island with unknown function. We decided to use this well-characterized myotube system, where a negative impact of Nctc1 promoter activity on $\mathrm{H} 19$ transcription has been demonstrated $^{36}$, to test the hypothesis that the SAHH-H19 interaction may affect CME methylation, which in turn influences the transcriptional activity of $\mathrm{Nctc1}$ and $\mathrm{H} 19$.

Thus, we downregulated $\mathrm{H} 19$ using an H19-specific short interfering RNA (siRNA; siH19 (refs 16,29)) in myotubes differentiated from mouse myoblasts, followed by in vivo SAHH activity assessment using homocysteine as a readout ${ }^{37}$. When the $H 19$ level was reduced to $\sim 20 \%$ of the control (Fig. 2a, left column) without significantly affecting the level of SAHH mRNA (right column), an $\sim 20 \%$ increase in SAHH activity was observed (Fig. 2b). These results supported the view that H19 association inhibits SAHH's ability to hydrolyse SAH, a strong inhibitor of SAM-dependent methyltransferases including DNMTs. We hypothesized that increased SAHH activity would activate DNMTs, which otherwise would be inhibited by SAH, leading to increased gene methylation. Indeed, we observed a 30\% increase in a differentially methylated region (DMR) within the CME as determined by quantitative methylation-specific PCR (QMSP) analysis (Fig. 2c). The PCR primers were designed to cover this DMR based on our single-nucleotide resolution genome-wide DNA methylation analysis (Fig. 2d, and see below). Primers that cover another DMR (DMR-2) of CME showed similar results (Supplementary Fig. 3). Next, we wanted to test whether DNMT3B might be responsible for this increased methylation. It was previously reported that in human skeletal muscle cells the enzymatic activity (but not the protein level) of DNMT3B increased in response to free fatty acids, resulting in promoter hypermethylation of $P G C-1 a$ (ref. 38). Further, recent genome-wide DNA methylome studies revealed a strong association of DNMT3B with gene body methylation and its positive correlation with gene expression ${ }^{1,39}$. Thus, CME methylation was evaluated in the presence of double siRNA knockdown. In the H19/Dnmt3b double knockdown group, Dnmt3b was silenced by $\sim 40 \%$ at the RNA (Fig. 2e, right column) and $\sim 70 \%$ at the protein levels (Fig. 2f, top blot). While H19 single knockdown expectedly increased CME methylation (Fig. 2g, compare middle bar with left bar) without altering the DNMT3B protein level (Fig. 2f, top blot, compare middle lane with left lane), the double knockdown restored methylation to the control level (Fig. 2g), suggesting that DNMT3B contributed to increased CME methylation induced by $H 19$ knockdown. A second siRNA (siDnmt3b-2) targeted to a different region of Dnmt3b produced similar results (Supplementary Fig. 4), confirming the specificity of the Dnm3b siRNA effects. Further, we carried out chromatin immunoprecipitation (ChIP) analysis using a DNMT3B antibody shown to be highly specific for DNMT3B in a genome-wide ChIP study ${ }^{1}$. Results revealed no significant change in DNMT3B occupancy at the DMR when H19 was downregulated (Fig. $2 \mathrm{~h}$ ), although a readily detectable change in RNAP association with $\mathrm{Nctcl}$ was observed under the same conditions (see below). These results suggested that it was the activity and not the chromatin residence of DNMT3B that was responsible for the methylation change. This result is in line with a previous report showing that a change in the enzymatic activity of DNMT3B alone was sufficient to induce hypermethylation of $P G C-1 a$ in human skeletal muscle cells ${ }^{38}$. Collectively, these results suggested that reducing $\mathrm{H} 19$ level de-represses SAHH and enables it to act on SAH, thereby relieving inhibition of DNMT3B by SAH and allowing DNMT3B to methylate CME.

CME methylation correlates with enhanced Nctc1 transcription. To determine the effect of CME methylation on Nctc1 transcription, we performed ChIP analysis using an anti-Ser-5(P)-RNAP antibody that specifically recognizes activated RNAP as previously described $^{35,36}$. We compared binding of activated RNAP to Nctc1 between control siRNA (siCon)- and siH19-transfected myotubes to assess relative transcription activity of $N c t c 1$ (refs 35,36 ). We observed an approximately fourfold enrichment in RNAP at Nctcl in siH19- versus siCon-transfected myotubes (Fig. 2i), suggesting an increase in Nctcl transcription that was further supported by the concomitant increase in the Nctcl RNA level (Fig. 2j). This positive correlation between CME methylation and Nctc1 transcription was not surprising because increased gene body methylation has been associated with increased transcription $^{1,3,39,40}$. We further noted a significant decrease in RNAP association with $\mathrm{H} 19$ in siH19-transfected myotubes (Fig. 2i, middle column, compare right green bar with left green bar). These results recapitulated the previously reported observation showing an inverse relationship of transcriptional activity between Nctc1 and H19 (ref. 36) and suggested a novel functional role of CME methylation in the regulation of Nctc1 and H19.

SAHH is required for H19-dependent CME methylation change. To provide further evidence that increased CME methylation induced by $H 19$ knockdown was indeed mediated by SAHH, double knockdown experiments using a Sahh-specific siRNA ${ }^{10}$ were carried out. In the H19/Sahh double knockdown cells, Sahh was silenced by $\sim 60 \%$ at both the RNA (Fig. 3a, right column) and protein levels (Fig. 3b, top blot). While H19 single knockdown increased methylation, H19/Sahh double knockdown abrogated this effect (Fig. 3c). The abrogation effect was also observed when a pharmacological SAHH inhibitor D-Eritadenine (DEA; Supplementary Fig. 5) ${ }^{41,42}$ was used (Fig. 3c). These results further supported the notion that SAHH is required for H19-mediated CME methylation change.

As increased CME methylation following $H 19$ knockdown correlated with increased Nctc1 transcription (Fig. 2g-j), we predicted that $\mathrm{H} 19$ and Sahh double knockdown would reverse this effect, given that increased CME methylation was dependent on SAHH activation (Fig. 3c). Consistent with our earlier observations (Fig. 2i, left column; and Fig. 2j, right column), H19 single knockdown increased RNAP association with Nctcl (Fig. 3d, left column, compare middle green bar with left green bar) with a concomitant increase in the Nctcl RNA level (Fig. 3e, compare middle bar with left bar). In the H19 and Sahh double knockdown cells, RNAP association with Nctc1 was significantly reduced compared with that in the H19 single knockdown cells (Fig. 3d, left column, compare right green bar with the middle green bar), and there was a decrease in the Nctcl RNA level as well (Fig. 3e). Moreover, the inverse relationship of RNAP association between Nctcl and $\mathrm{H} 19$ was lost in the double knockdown cells (Fig. 3d, compare between the left and middle columns). Collectively, these results strongly suggested that $\mathrm{H} 19$ induced CME methylation change through the action of SAHH, which in turn affected the transcription of Nctcl and H19.

U-rich element binding inhibits SAHH activity in vitro. To further delineate the molecular nature of SAHH-H19 interaction, we took an in vitro approach to map binding sites of SAHH on H19. Both GAPDH and LDH bind to AU- and U-rich RNA elements via the Rossmann-fold ${ }^{32,33}$. SAHH has a Rossmann-fold that binds cofactor $\mathrm{NAD}^{+}$, which is required 

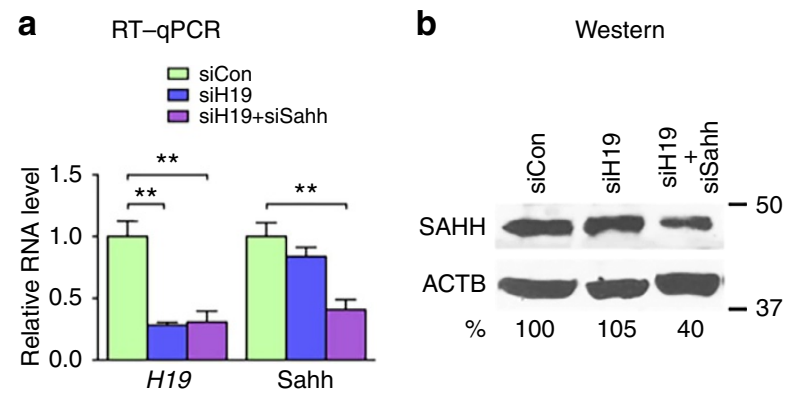

d

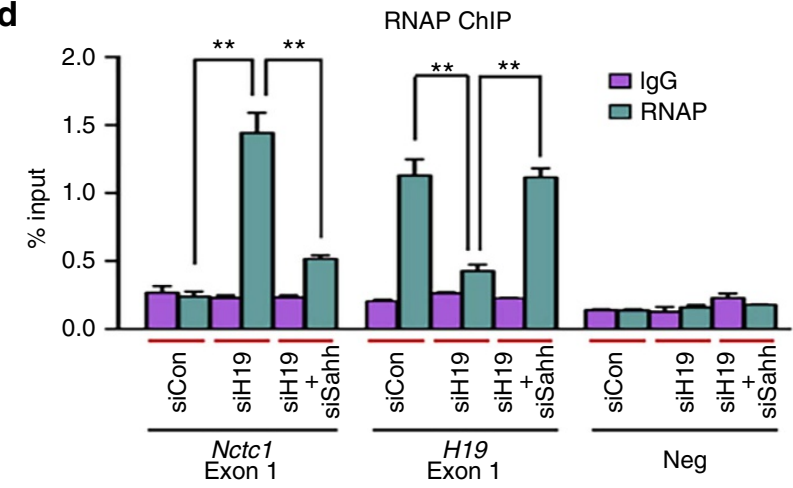

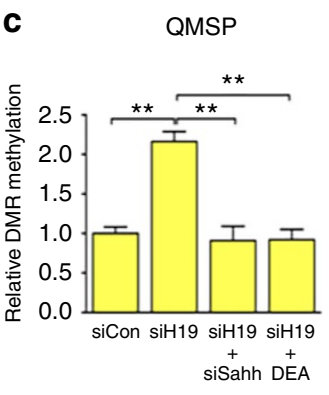

e

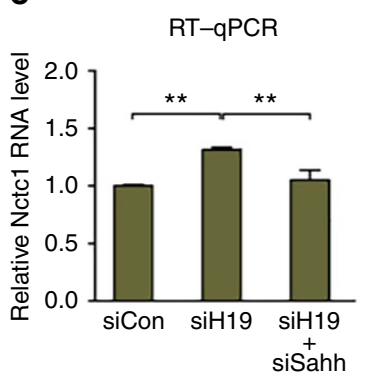

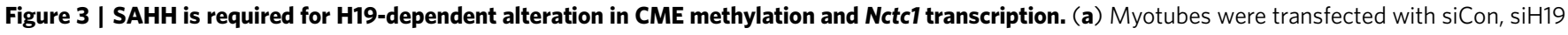

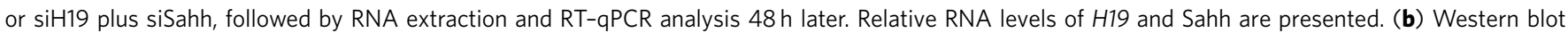

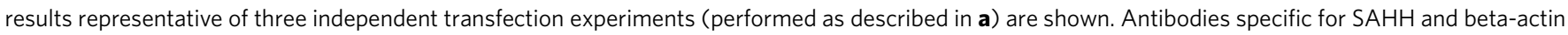
were used on the top and bottom blots, respectively. Numbers indicate SAHH protein levels after normalization against beta-actin loading controls with levels in siCon cells arbitrarily set as $100 \%$. (c) Myotubes were transfected with siCon, siH19, siH19 plus siSahh or siH19 plus incubation with $10 \mu \mathrm{M}$ of DEA $20 \mathrm{~h}$ post transfection. DMR methylation was assessed $48 \mathrm{~h}$ post transfection using QMSP. (d) Association of activated RNAP at Nctc1 and H19. Myotubes were transfected with siCon, siH19 or siH19 plus siSahh. ChIP analysis was performed $48 \mathrm{~h}$ post transfection. (e) Relative Nctc1 RNA levels measured $48 \mathrm{~h}$ post transfection, as described in $\mathbf{d}$. Student's $t$-tests were performed to compare differences between quantitative variables. Numbers are mean \pm s.d. $(n=3) .{ }^{\star \star} P<0.01$.

for maintaining its quaternary structure and catalytic function ${ }^{8}$. We hypothesized that SAHH may bind U-rich elements (URE), as we noticed stretches of UREs flanked by $\mathrm{C}$ or $\mathrm{G}$ near the 3 '-end of both human and mouse H19 (Fig. 4a). To test this hypothesis, we performed electrophoretic mobility shift assays (EMSAs) using purified recombinant human SAHH protein (rSAHH) and $3^{\prime}$-end biotin-labelled H19 RNA fragments. At low rSAHH concentrations, only one complex (complex 1) formed with both human (hURE) and mouse (mURE) fragments (Fig. 4b, top two panels on the left). As rSAHH concentration rose, an additional complex (complex 2) appeared, suggesting simultaneous binding of more than one molecule of rSAHH to multiple U-rich sequences. We estimated the $K_{\mathrm{d}}$ values of rSAHH binding to the human and mouse UREs to be 0.44 and $0.46 \mu \mathrm{M}$, respectively (Fig. $4 \mathrm{~b}$, bottom panel), similar to the $K_{\mathrm{d}}$ value of LDH for the granulocyte-macrophage colony-stimulating factor RNA AU-rich element, which was reported to be $0.5 \mu \mathrm{M}$ (ref. 33). The apparently slightly lower $K_{\mathrm{d}}$ value of hURE was also reflected by competition experiments showing that unlabelled hURE competed better for binding to rSAHH than the unlabelled mURE (Fig. 4c, left two panels). A non-U-rich fragment (hNSE) randomly chosen from human $\mathrm{H} 19$ failed to efficiently compete for rSAHH binding (Fig. 4c, third panel), which was consistent with its poor rSAHH-binding affinity (Fig. 4b). Further, the poly(U) ribohomopolymers exhibited the highest activity in reducing complex formation (Fig. 4c, fourth panel). Together, these results showed that purified rSAHH was capable of direct binding to UREs in both human and mouse $\mathrm{H} 19$ in vitro.
As $H 19$ binds and inhibits SAHH activity in vivo (Figs $1 \mathrm{c}, \mathrm{d}$ and 2b), we tested effects of H19 fragments on the ability of $\mathrm{rSAHH}$ to hydrolyse $\mathrm{SAH}$ in vitro. Both hURE and mURE inhibited the hydrolytic activity of $\mathrm{rSAHH}$ in a dose-dependent manner, whereas hNSE did not (Fig. 4d). This was in line with the observation that both UREs had significantly higher affinity for rSAHH than hNSE (Fig. 4b,c). As expected, poly(U) also efficiently inhibited rSAHH activity (Fig. 4d). Combined with our in vivo data (Figs 1c,d and 2b), these results strongly support our hypothesis that $\mathrm{SAHH}$ binding to $H 19$ interferes with its ability to hydrolyse $\mathrm{SAH}$ in vivo.

H19-dependent SAHH inhibition exerts global effects. As SAHH can potentially affect SAM-dependent methyltransferases other than DNMT3B, one would predict that H19 knockdown would lead to global DNA methylation changes. Methylation at a particular cytosine residue within a gene locus is influenced both by the activity of DNMTs and by other epigenetic marks that affect their accessibility ${ }^{1-3}$. SAHH activity change could not only directly affect the activity of DNMTs, but also other epigenetic marks, which in turn might indirectly affect gene methylation. Further, not all methyltransferases display an equal sensitivity to SAHH inhibition ${ }^{8}$. Thus, increased SAHH activity as a result of H19 knockdown is expected to produce three different outcomes: some genomic loci would become hypermethylated; some hypomethylated; and some would not change, relative to control knockdown. 
a H19URE sequences

hURE (nt 1,889-2,016)

CUUCUUUUUCAUCCUUCUGUCUCUUUGUUUCUGAGCUUUCCUGUCUUUCCUUUUUUCUGAGAGAUUC AAAGCCUCCACGACUCUGUUUCCCCCGUCCCUUCUGAAUUUAAUUUGCACUAAGUCAUUUG

mURE (nt 2,201-2,322)

CCUGUCUUUUUCUUCUUCCUCCUUUCCUGUAAUUCUGUUUCUUUCCUUUUGUUCCUUCCUUGCUUGA GAGACUCAAAGCACCCGUGACUCUGUUUCCCCAUUUACCCCCUUUUGAAUUUGCA

hNSE (nt 1,013-1,143)

CUGGUGCGGAGAGGGCCCACAGUGGACUUGGUGACGCUGUAUGCCCUCACCGCUCAGCCCCUGGGG

CUGGCUUGGCAGACAGUACAGCAUCCAGGGGAGUCAAGGGCAUGGGGCGAGACCAGACUAGGCGA

b

EMSA

hURE binding

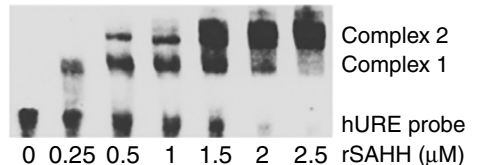

mURE binding

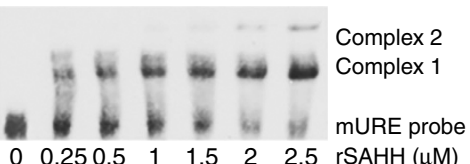

hNSE binding

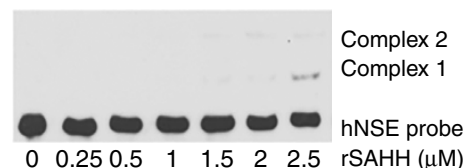

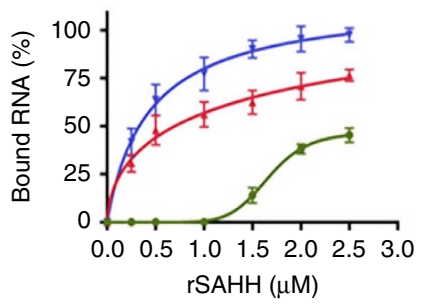

$\rightarrow$ hURE $K_{\mathrm{d}}=0.44, R^{2}=0.97$

- mURE $K_{\mathrm{d}}=0.46, R^{2}=0.95$

$\rightarrow$ hGRE $K_{\mathrm{d}}=1.66, R^{2}=0.98$

C

EMSA competition

Unlabelled RNA:

hURE

Molar excess:

$4 \times 8 \times 16 \times 32 \times$

MURE

hNSE

$4 \times 8 \times 16 \times 32 \times$

$4 \times 8 \times 16 \times 32 \times$

Poly(U)

Complex 2

hURE probe

Fraction bound (\%)
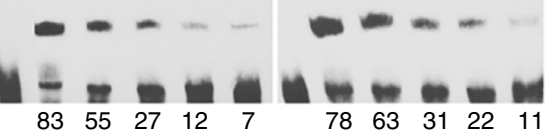

$\begin{array}{lllll}78 & 63 & 31 & 22 & 11\end{array}$

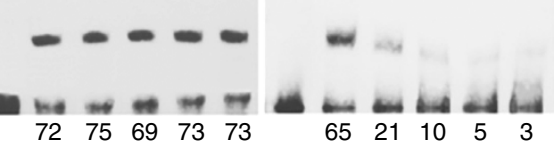

d

In vitro SAHH activity inhibition
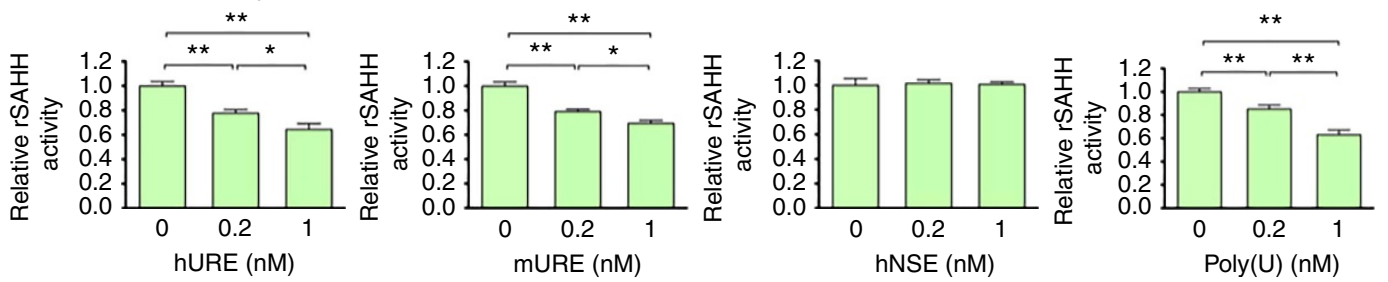

Figure 4 | Binding to U-rich elements of H19 inhibits SAHH activity in vitro. (a) Sequences of human and mouse H19 fragments used in EMSA and in vitro SAHH activity assays, with the U-rich stretches underlined. Numbers in parentheses mark the nucleotide positions of the sequences relative to the transcription start sites of H19. (b) EMSA results using biotin-labelled hURE, mURE or hNSE with increasing concentrations of $r S A H H$. The positions of unbound RNA and RNA-protein complexes are marked to the right. Band intensities were quantified from three independent experiments and used to calculate the $K_{d}$ values displayed in the bottom panel. (c) EMSA results with biotin-labelled hURE and $2 \mathrm{mM}$ of $r S A H H$. The amounts of unlabelled competitors added in relative molar excess are indicated on top. Band intensities were calculated and presented as percentage of fraction bound. Numbers are average of two independent experiments. (d) In vitro $\mathrm{rSAHH}$ activity assay showing dose-dependent inhibition of the hydrolytic activity of $r \mathrm{SAHH}$ by the UREs and poly $(U)$. Student's $t$-tests were used to compare differences between quantitative variables. Numbers are mean \pm s.d. $(n=4)$. ${ }^{\star \star} P<0.01$, ${ }^{\star} P<0.05$.

As a proof-of-principle that $H 19$ modulates gene methylation through SAHH, we performed global DNA methylation studies to identify genes whose methylation might be affected in a way similar to that of Nctc1. Mytotubes were transfected with siH19 (or siCon as a control), and DNA was extracted $48 \mathrm{~h}$ later and subjected to genome-scale DNA methylation mapping using the platform of an improved version of Reduced Representation
Bisulfite Sequencing. This platform offers detection of - three to four million unique $\mathrm{CpG}$ sites, allowing $>85 \%$ coverage of all annotated $\mathrm{CpG}$ islands and $>80 \%$ of all gene promoters for a maximal amount of methylation data. It allows the identification and analysis of DMRs between samples at single-nucleotide resolution. As expected, following $\mathrm{H} 19$ knockdown we observed extensive genome-wide methylation pattern changes relative to 
siCon cells, with some genes showing increased methylation, others showing decreased methylation and a third group with no significant change (Supplementary Fig. 6). We hypothesized that in the group of genes showing increased methylation, some were likely affected by increased activity of DNMT3B as a direct result of $\mathrm{SAHH}$ activation. To test this possibility, we selected five genes showing strong hypermethylation with siH19 (Supplementary Data 1). Since SAHH dysfunction is associated with human diseases $8,10,11$, we selected genes having important cellular functions or implicated in diseases. $B m p 8 b$ (bone morphogenetic protein $8 \mathrm{~b}$ ) has been reported to act as a signalling molecule in energy balance regulation in both brown adipocytes and neurons, and $B m p 8 b$ null mice exhibit decreased rate of metabolism and impaired thermogenesis ${ }^{43}$. Casp2 (Caspase 2) is a member of the caspase family that mediates cellular apoptosis through the proteolytic cleavage of specific protein substrates ${ }^{44}$. In addition to its well-defined role in cell death, Casp2 has been implicated in neurodegenerative diseases, tumorigenesis, metabolism and aging ${ }^{44}$. Cdt1 (chromatin licensing and DNA replication factor 1 ) is a component of the prereplicative complex that assembles on genomic DNA at origins of replication. Mutations in this gene have been associated with growth failure in primordial dwarfism $^{45}$. Foxa2 (forkhead box A2) is a DNA-binding transcription factor essential for the development of endodermderived organs including the liver and pancreas. Foxa2 has been implicated in blood glucose homeostasis by controlling the expression of multiple genes in pancreatic beta-cells ${ }^{46}$. Setd 2 (SET domain containing 2) is a member of the SAM-dependent histone lysine methyltransferase family (which also includes $\mathrm{EZH} 2$, a component of PRC2) known to be important for chromatin modification ${ }^{2,47}$. SETD2 interacts with the Huntington's disease (HD) protein Huntingtin and has been implicated in the pathogenesis of $\mathrm{HD}$, a neurodegenerative disorder characterized by loss of striatal neurons ${ }^{48}$.

To determine whether the increased methylation in these genes as revealed by genome-wide analysis was SAHH-dependent, myotubes were transfected with siH19 or siCon, with or without co-transfection of siSahh or treatment of SAHH inhibitor DEA. QMSP analysis was carried out $48 \mathrm{~h}$ post transfection using primers that specifically amplify a single DMR of each respective gene (Fig. 5a). While H19 knockdown increased DMR methylation, its combination with siSahh or DEA rescued methylation to the level of the control (Fig. 5b). These results strongly suggested H19-mediated, SAHH-dependent regulation of methylation at these sites, thus validating our genome-wide data. To further address whether DNMT3B might contribute to the increased methylation, H19/Dnmt3b double knockdown experiments were carried out, followed by QMSP analysis. As shown in Fig. 5c, siH19 in combination with either Dnmt3b siRNA was able to partially or fully restore methylation to the level of the siCon control. The partial rescue by the Dnmt3b siRNAs suggested that other DNMTs may contribute to the methylation of these sites, which warrants future investigation. It is noteworthy that, while a single DMR methylation change may seem small, the combined effects from multiple DMRs in a single gene (Supplementary Table 1 and Gene Expression Omnibus (GEO) accession number GSE73014) could potentially be synergistic, leading to bigger biological effects.

Next, DNMT3B ChIP analysis was conducted following siRNA transfection (Fig. 6a). While no DNMT3B association was detected at the DMRs of Cdt1 and Setd2, for the remaining three genes tested, no change in DNMT3B occupancy at the respective DMRs was observed following H19 knockdown (Fig. 6b). These results supported the notion that the $H 19$ knockdown-induced increase in methylation was due to changes in DNMT3B activity rather than its chromatin association.
H19 regulates gene expression via multiple mechanisms, both in the nucleus and in the cytoplasm. Thus, H19 knockdown could alter its nuclear interaction with methyl-CpG-binding domain protein 1 and/or EZH2 to affect gene transcription ${ }^{23,27}$. In the cytoplasm, H19 knockdown could change the dynamic association of $\mathrm{K}$ homology-type splicing-regulatory protein with its target mRNAs, leading to their stability changes ${ }^{28}$. The mRNA levels of genes targeted by let- 7 could also be affected, as H19 acts as a molecular sponge for let-7 (ref. 29). Finally, H19 could modulate transcription by affecting gene methylation through interaction with SAHH (this report). Thus, the effects of H19 knockdown on genome-wide transcript levels would represent a summation of contributions from the different mechanisms outlined above as well as from other yet unknown mechanisms. Consistent with this view, our RNA-sequencing (RNA-seq) analysis revealed profound changes in transcript profiles between siCon- and siH19-transfected myotubes (Supplementary Data 2 and GEO accession number GSE73014). While a number of these are consistent with alterations in DNA methylation profiles, the multiplicity of $\mathrm{H} 19$ functions does not at this time allow definitive mechanistic connections to be established.

\section{Discussion}

This study uncovers a novel regulatory circuit of gene methylation dictated by the interaction between $\mathrm{H} 19$ and SAHH. Using the Igf2-H19-Nctc1 gene locus in mouse muscle cells as a model, we demonstrate that binding of SAHH to H19 interferes with its ability to hydrolyse $\mathrm{SAH}$, which is a potent product inhibitor of SAM-dependent methyltransferases. This leads to decreased DNMT3B-mediated methylation within the Nctc1 gene body and subsequently reduced transcriptional activity of the Nctc1 promoter. We show that UREs in H19 that display preferential binding to SAHH also inhibit the catalytic activity of SAHH, suggesting that the basis for this preferential interaction is recognition of the UREs. Further, we demonstrate that this H19SAHH-dependent regulation occurs at other genomic loci, suggesting global effects of this regulation. On the basis of these findings, we propose the model illustrated in Fig. 6c. To our knowledge, this represents the first case in which a single lncRNA acts in trans to alter the epigenetic landscape in a genome-wide manner.

Importantly, our findings offer novel insights into DNA methylation regulation associated with development and pathological conditions. Specifically, our results suggest that the enzymatic activity of chromatin-bound DNMT3B might be subjected to modification by $H 19$ through the H19/SAHH pathway. Interestingly, DNMT3B (but not DNMT1) was shown to be required for restoring demethylated genomic regions induced by the methylation inhibitor 5-Aza-CdR (ref. 39). Further, a recent genome-scale study comparing DNMT occupation with DNA methylation patterns showed that a fraction of gene loci bound by DNMTs does not overlap with methylation and that the number of such loci changes dramatically on cell differentiation ${ }^{1}$. It has been proposed that loci bound by DNMTs, but which are not methylated, may be primed for later de novo methylation events that are associated with cell pluripotency and differentiation states ${ }^{1}$. It is possible that the bound DNMTs are inactive because of inhibition (by yet unknown mechanisms), which is removed as cell states change. H19 expression is both temporally and spatially regulated during development, and its aberrant expression has been linked to human genetic disorders and cancer ${ }^{14,22}$. It is tempting to postulate that the enzymatic activity of DNMTs might be modulated in response to developmental cues and pathological 

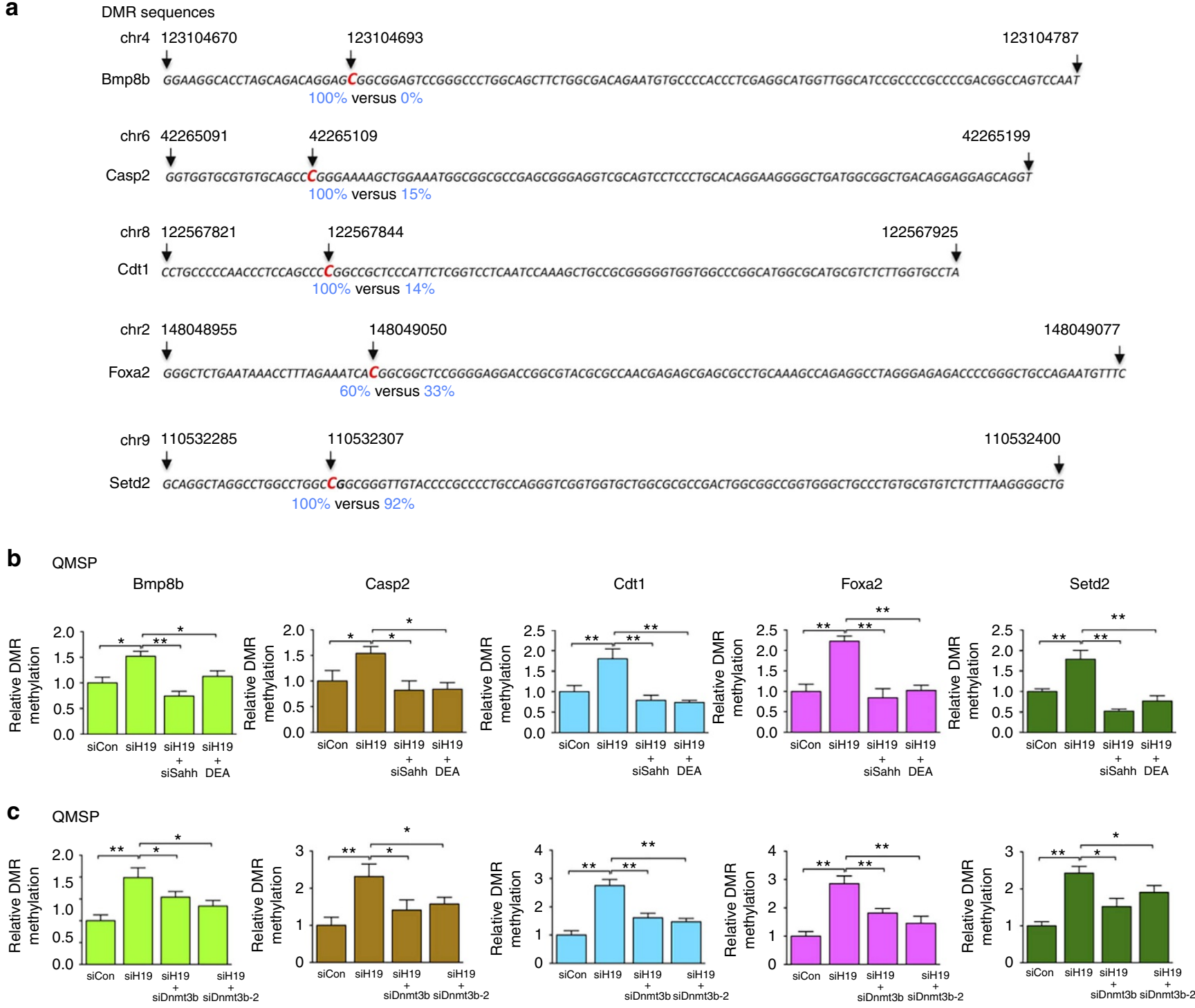

Figure 5 | SAHH is required for H19-dependent methylation change in the DMRs of other genes. (a) Sequences of the DMRs of the indicated genes amplified using QMSP primers. The differentially methylated cytosine residues are highlighted in red. Numbers above the sequences mark the positions of the indicated nucleotides in the chromosomes. Numbers below (in blue) indicate percentage of methylation in the siH19 (left) versus siCon (right) cells as determined by genome-wide methylation mapping. (b) Myotubes were transfected with siCon, siH19, siH19 plus siSahh or siH19 plus incubation with $10 \mu \mathrm{M}$ of DEA $20 \mathrm{~h}$ post transfection. DNAs were isolated $48 \mathrm{~h}$ post transfection and analysed using QMSP. (c) Myotubes were transfected with siCon, siH19, siH19 plus siDnmt3b or siH19 plus siDnmt3b-2. DNAs were isolated $48 \mathrm{~h}$ later and analysed using QMSP. Student's $t$-tests were used to compare differences between quantitative variables. Numbers are mean \pm s.d. $(n=3)$. ${ }^{\star} P<0.01,{ }^{\star} P<0.05$.

conditions and that the H19-SAHH axis might be a functional link underlying these regulations.

It has become increasingly evident that the relationship between methylation and gene expression is far more complex than previously thought. Unlike the classic models linking promoter methylation to gene silencing and gene body methylation to expression, emerging models posit that the correlation between methylation and gene expression is genomic contextdependent $t^{1,3}$. Factors such as distance of CpGs relative to the transcription start site, their residence in $\mathrm{CpG}$ islands, promoters, enhancers or gene bodies as well as combinations of any of these can positively or negatively affect gene expression. Thus, it is not merely the sum or density of all methylated CpGs (which were used to label a gene as being 'hyper' or 'hypo' methylated) but rather the combinations of the various factors described above as well as of other yet unknown factors that determine the outcome on gene expression. Our studies reveal that H19 regulates methylation at many genomic sites through modulating the activity of SAHH. Further investigation will certainly unravel a highly complex and context-dependent nature of this regulation. Depending on cell/tissue types, developmental stages and disease states, the biological end points of this H19/ SAHH-mediated regulation of gene methylation are likely to be diverse. Curiously, the brain expresses very low or undetectable levels of $H 19$ (ref. 18) while accumulating uniquely high levels of DNA hydroxymethylation (5-hydroxymethylcytosine) during postnatal development ${ }^{49}$. Hydroxymethylation occurs through oxidation of the methyl group of 5-methylcytosines by TET enzymes, and this epigenetic modification is thought to be important for neuronal differentiation and function ${ }^{49-51}$. As hydroxymethylation has been implicated in the active DNA demethylation mechanism, it remains to be determined whether the activity of SAHH in the brain might be significantly lower or the activity of SAHH might be subjected to regulation by 
a $\mathrm{RT}-\mathrm{qPCR}$

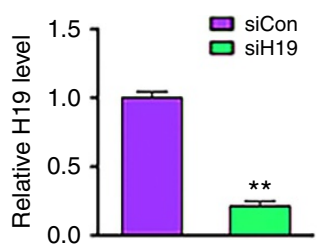

C Model
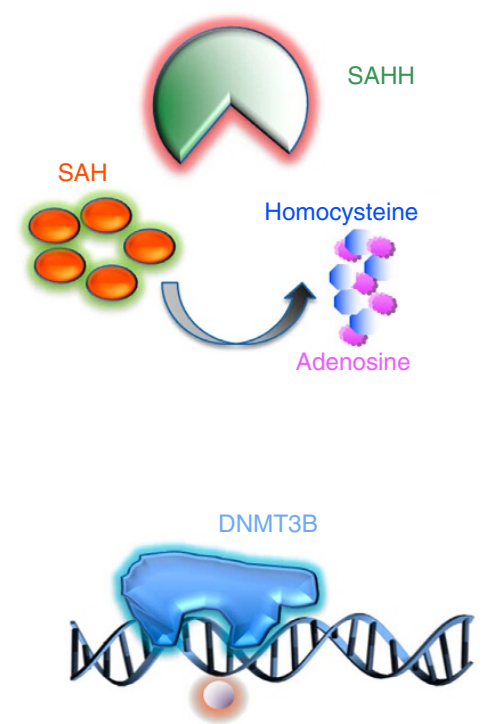

b DNMT3B ChIP

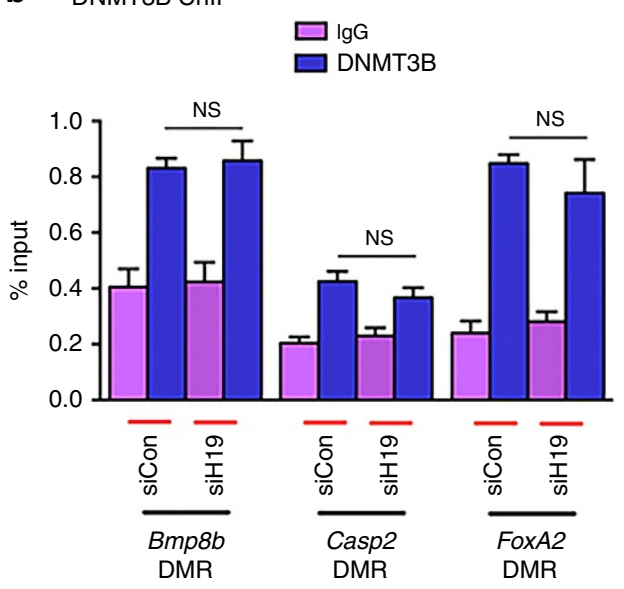

$\mathrm{CH}_{3}$

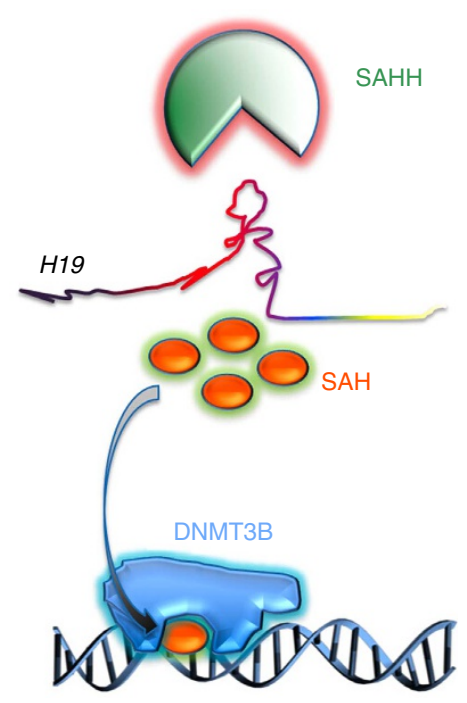

Figure 6 | A model for H19/SAHH-dependent regulation of gene methylation. (a) Myotubes were transfected with siCon or siH19, followed by RNA extraction and RT-qPCR analysis $48 \mathrm{~h}$ later. Relative $H 19$ level is presented. Student's $t$-tests were used to compare differences between quantitative variables. Numbers are mean \pm s.d. $(n=3) .{ }^{*} P<0.01$. (b) DNMT3B ChIP at the DMRs of the indicated genes. ns, not statistically different. (c) In the absence of $\mathrm{H} 19$ (left panel), SAHH hydrolyses SAH to homocysteine and adenosine, and DNMT3B is active in DNA methylation. When H19 is present (right panel), SAHH activity is attenuated because of its association with H19. This leads to accumulation of SAH, which binds to DNMT3B and prevents it from methylating DNA.

non-H19-mediated mechanisms. Finally, as SAM-dependent methyltransferases methylate other cellular components besides DNA, our findings open up new avenues for future investigations of other cellular components that might be regulated by the $\mathrm{H} 19$ / SAHH-dependent mechanism.

\section{Methods}

Reagents and plasmids. Mouse monoclonal anti-SAHH (Santa Cruz Biotechnology, sc-271389), rabbit polyclonal anti-SAHH (Proteintech, 10757-2-AP), mouse monoclonal anti-HuR (Santa Cruz, sc-5261), rabbit polyclonal anti-HuR (Proteintech, 11910-1-AP), rabbit polyclonal anti-DNMT3B (Novus, NB300-516), rabbit polyclonal anti-RNAP CTD repeat YSPTSPS (phospho S5; Abcam, ab5131), rabbit polyclonal anti- $\beta$-tubulin (Abcam, ab6046), mouse monoclonal anti- $\beta$-actin (Abcam, ab8226), mouse pre-immune IgGs (Chemicon, PP54) and rabbit pre-immune IgGs (Thermo Scientific, Prod \#1862244) were purchased. SiRNAs specific for mouse H19 (siH19, 4390816/n253566), SAHH (siSahh, AM16708/ 72248), Dnmt3b (siDnmt3b, AM16708/161533; siDnmt3b-2, 4390771/S65080) and negative siCon (AM4636) were purchased from Ambion/Life Technologies. The SAHH inhibitor DEA (sc-207632) was purchased from Santa Cruz. To construct
pH19, PCR was carried out using human term placental cDNA as a template with forward $5^{\prime}$-aaggaaaaaagcggccgcAGCAGGGTGAGGGAGGGGGTG-3 ${ }^{\prime}$ and reverse $5^{\prime}$-cgcggatccGTAACAGTGTTTATTGATGATGAGTC- $3^{\prime}$ primers. The lower-case letters represent restriction enzyme sites for NotI and BamHI, respectively, and the upper-case letters are complementary to $\mathrm{H} 19$ sequence. The resulting 2.6-kb-long PCR product was inserted into pFLAG-CMV-2 (Sigma) opened with NotI and BamHI. To make pH19-S1, an annealed oligonucleotide fragment containing a 44-nt-long S1 sequence ( $5^{\prime}$-ACCGACCAGAATCATG CAAGTGCGTAAGATAGTCGCGGGCCGGG-3') was cloned into $\mathrm{pH} 19$ at its $3^{\prime}$-end opened with BamHI. pS1 was made by inserting the S1 sequence into pFLAG-CMV-2 (Sigma) opened with NotI and BamHI.

Cell culture and transfection. HEK293 cells (ATCC, CRL-1573) were cultured using standard protocols provided by the ATCC. Undifferentiated mouse C3H myoblasts (Sigma-Aldrich, 91031101-iVL) were maintained in growth medium (GM; DMEM, Gibco, 11965-092, supplemented with 10\% fetal bovine serum, heatinactivated, $1 \%$ penicillin/streptomycin, $1 \% \mathrm{~L}$-glutamine and 1-mM sodium pyruvate). To prepare for differentiation, cells were seeded at a density of $1.6 \times 10^{5}$ or $1.0 \times 10^{4}$ cells per well in GM in 6- or 48-well plates, respectively. Differentiation was initiated 2 days later when cells became confluent by replacing GM with 
differentiation medium containing $2 \%$ horse serum in place of $10 \%$ fetal bovine serum. The medium was changed every other day until transfection, which was performed on days 3-4 after initiation of differentiation.

For cell transfection, mytotubes were washed once with pre-warmed OPTI-MEM immediately before addition of transfection cocktails. To prepare transfection cocktails for each well of six-well plates, 250 pmol of siCon or siH19 (with a stock solution of $5 \mu \mathrm{M}$ ) was mixed with $300 \mu \mathrm{l}$ of OPTI-MEM by gentle pipetting. In parallel, $12.5 \mu \mathrm{l}$ of Lipofectamine 2000 was diluted in $300 \mu \mathrm{l}$ of OPTI-MEM by gentle pipetting. After 5 -min incubation at room temperature, the two were combined by gentle pipetting. Following incubation at room temperature for $25 \mathrm{~min}$, the resulting cocktail $(600 \mu \mathrm{l})$ was directly applied to myotubes that were pre-washed with OPTI-MEM. After overnight incubation in a tissue culture incubator, the cocktail was replaced with fresh differentiation medium. Forty-eight hours later, RNA and protein were extracted for analysis.

RNA in situ hybridization and SAHH immunofluorescence. Undifferentiated myoblasts were seeded in $\mu$-Slide eight-well plates (Ibidi cat. no. 80826) at $\sim 2 \times 10^{4}$ cells per well, and differentiation was initiated as described above. H19 RNA FISH experiments were carried out using the RNAscope fluorescent Multiplex system (Advanced Cell Diagnostic Inc.) according to the manufacturer's instructions. The protocol involves proteinase $\mathrm{K}$ treatment to remove proteins including nucleases. The system included the following components: Probe-Mm-H19-C3 (P/N: 423751-C3, L/N: 14241A; custom-designed specific for mouse H19), Blank-C1 (P/N: 300041; L/N: 14132A), 3-Plex Negative Control probe (P/N: 320371; L/N: 14105A), Pretreatment Kit-FL FF (P/N: 320842; L/N: 14099A) and Detection Kit-FL (P/N: 320851; L/N: 14098A). For SAHH immunostaining, myotubes were washed briefly with TBS $(20 \mathrm{mM}$ Tris- $\mathrm{HCl}$, pH 7.4, $225 \mathrm{mM} \mathrm{NaCl}$ ), fixed with $3 \%$ paraformaldehyde/TBS for $20 \mathrm{~min}$, permeabilized with $1 \%$ SDS/TBS for 5 min and blocked with $10 \% \mathrm{BSA} / 0.1 \%$ goat serum/TBS for $1 \mathrm{~h}$. Cells were then incubated with polyclonal anti-SAHH antibody at a dilution of 1:100 for $1 \mathrm{~h}$, washed with TBS and then incubated with Alexa-488 secondary antibody (1:500 dilution, Invitrogen, Grand Island, NY) for $1 \mathrm{~h}$. After washing with TBS, cells were mounted with mounting medium containing 4,6-diamidino-2-phenylindole (Vector Lab, Burlingame, CA). Images were obtained under the fluorescence microscopy (Axiovert S 100; Carl Zeiss, Oberkochen, Germany).

RNP affinity purification and protein identification. HEK293 cells were transfected with pH19-S1, pH19 or pS1 in a $25-\mathrm{cm}$ plate scale and harvested $48 \mathrm{~h}$ later by manual scraping and pelleted by centrifugation. After washing twice in ice-cold PBS, cell pellet was resuspended in $1.2 \mathrm{ml}$ of ice-cold gentle lysis buffer (GLB) (10 mM Tris-HCl, pH 7.5; $10 \mathrm{mM} \mathrm{NaCl}$; $10 \mathrm{mM}$ EDTA; 0.5\% Triton X-100; $1 \mathrm{mM}$ phenylmethylsulphonyl fluoride (PMSF), $1 \times$ proteinase inhibitor cocktail (CalBiochem, 539137); $1 \mathrm{mM}$ dithiothreitol (DTT) and $1 \times$ RNase inhibitor (Roche, 10412600)) in a 1.5-ml eppendorf tube and incubated on ice for $20 \mathrm{~min}$. Cell lysate was centrifuged to remove insoluble materials. The lysate was precleared by incubation at $4{ }^{\circ} \mathrm{C}$ for $1.5 \mathrm{~h}$ with $40 \mu \mathrm{l}$ of avidin agarose beads (Pierce, 20219) with $\mathrm{NaCl}$ added to a final concentration of $150 \mathrm{mM}$. The cleared lysate was mixed with $40 \mu \mathrm{l}$ of streptavidin beads (packed volume, Sigma, S1638-5ML) and the tube was rotated at $4{ }^{\circ} \mathrm{C}$ for $3 \mathrm{~h}$. The beads were collected and washed with $1 \mathrm{ml}$ of ice-cold GLB plus (10 mM Tris-HCl, pH 7.5; $150 \mathrm{mM} \mathrm{NaCl} ; 10 \mathrm{mM}$ EDTA; $0.5 \%$ Triton X-100) five times by rotating the tube at $4{ }^{\circ} \mathrm{C}$ for $3 \mathrm{~min}$ each. The purified RNPs were eluted from the beads in $20 \mu \mathrm{l}$ of $2 \times$ SDS sample buffer by heating at $10{ }^{\circ} \mathrm{C}$ for $5 \mathrm{~min}$ and resolved by $10 \%$ SDS-PAGE. Following Commassie staining, the protein band at $48 \mathrm{kDa}$ in the $\mathrm{pH} 19-\mathrm{S} 1$ lane was excised and submitted for mass spectrometry analysis by ProtTech Inc. (Norristown, PA 19403).

RIP analysis. To prepare antibodies, $20 \mu \mathrm{l}$ of protein A Sepharose beads were incubated with $20 \mu \mathrm{g}$ of monoclonal anti-SAHH antibody or $20 \mu \mathrm{g}$ of mouse pre-immune IgG in $500 \mu \mathrm{l}$ IP buffer $(0.5 \%$ Triton X-100, $200 \mathrm{mM} \mathrm{NaCl}, 10 \mathrm{mM}$ Tris- $\mathrm{HCl}$ at $\mathrm{pH} 7.5$ and $10 \mathrm{mM}$ EDTA) at $4{ }^{\circ} \mathrm{C}$ overnight. The next day, the beads were washed three times with IP buffer and kept on ice until used. To prepare cell lysates, myotubes (from one well of a six-well plate) were harvested and cell pellets resuspended in $600 \mu \mathrm{l}$ of freshly prepared lysis buffer $(0.5 \%$ Triton X-100, $10 \mathrm{mM}$ $\mathrm{NaCl}, 10 \mathrm{mM}$ Tris- $\mathrm{HCl}$ at $\mathrm{pH} 7.5,10 \mathrm{mM}$ EDTA, $0.5 \mathrm{mM}$ PMSF, $1 \mathrm{mM}$ DTT, $1 \times$ protease inhibitor cocktail (Calbiochem) and 400 units per ml RNase inhibitor). The suspensions were incubated on ice for $20 \mathrm{~min}$. After removing insoluble materials by centrifugation, lysates were precleared using $10 \mu \mathrm{l}$ of protein A sepharose ( $\mathrm{NaCl}$ was added to a final concentration of $200 \mathrm{mM}$ ), followed by addition of yeast tRNA (Ambion) to a final concentration of $40 \mu \mathrm{g} \mathrm{ml}^{-1}$. The cleared lysates were transferred to tubes containing antibody or pre-immune IgG-coated beads, and IP was carried out by rotating the tubes at $4{ }^{\circ} \mathrm{C}$ for $3 \mathrm{~h}$ Following IP, the beads were washed five times with IP buffer by adding $1 \mathrm{ml}$ of the buffer and rotating the tube at $4{ }^{\circ} \mathrm{C}$ for $2 \mathrm{~min}$ each time. RNA was extracted from the beads using the PureLink RNA Mini Kit (Ambion, cat. no. 12183018A). Reverse transcription was performed in a $40-\mu \mathrm{l}$ reaction volume using the Bio-Rad iScript cDNA synthesis kit, followed by quantitative PCR (qPCR).
Genomic DNA extraction. Genomic DNA was isolated using Quick-gDNA MicroPrep (Zymo, D3021) according to the manufacturer's instructions.

RNA extraction and RT-qPCR. Total RNAs were extracted from cells using the PureLink RNA Mini Kit (Ambion, cat. no. 12183018A). cDNA was synthesized using the Bio-Rad iSCRIPT kit (1725122) in a $20-\mu$ l reaction containing $100-500 \mathrm{ng}$ of total RNA. Quantitative real-time PCR (RT-qPCR) was performed in a $15-\mu \mathrm{l}$ reaction containing $0.5-1 \mu \mathrm{l}$ of cDNA using iQSYBRGreen (Bio-Rad) in a Bio-Rad iCycler. PCR was performed by initial denaturation at $95^{\circ} \mathrm{C}$ for $5 \mathrm{~min}$, followed by 40 cycles of $30 \mathrm{~s}$ at $95^{\circ} \mathrm{C}, 30 \mathrm{~s}$ at $60^{\circ} \mathrm{C}$ and $30 \mathrm{~s}$ at $72^{\circ} \mathrm{C}$. Specificity was verified using melting curve analysis and agarose gel electrophoresis. The threshold cycle $\left(C_{\mathrm{t}}\right)$ values of each sample were used in the post-PCR data analysis. The RT-PCR primers are listed in Supplementary Table 1.

Western blot analysis. Cell pellets were quickly lysed in five volumes of $2 \times$ SDS sample buffer heated at $100^{\circ} \mathrm{C}$ for $5 \mathrm{~min}$, with occasional vortexing. Five to ten microlitres of homogenized samples were loaded on 10\% SDS gel, followed by western blot analysis. The linear dynamic range of each protein of interest was determined by serial dilutions. Bands on western blot gels were quantified using ImageJ. Uncropped blots of Fig. 1c are shown in Supplementary Fig. 7.

QMSP analysis. Genomic DNA was extracted from myotubes in one well of six-well plates using Quick-gDNA MicroPrep. Fifteen microlitres of water were used to elute DNA from each column. For bisulfite treatment, 400-500 ng of DNA was used for each column using the EZ DNA Methylation-Gold Kit (Zymo, D5006). RT-qPCR was performed in a $15-\mu \mathrm{l}$ reaction containing $1 \mu \mathrm{l}$ of the eluant using iQSYBRGreen (Bio-Rad) in a Bio-Rad iCycler. The PCR primers (Supplementary Table 2) for methylated DNA were used at a final concentration of $0.3 \mu \mathrm{M}$ in each PCR reaction. PCR was performed by initial denaturation at $95^{\circ} \mathrm{C}$ for $5 \mathrm{~min}$, followed by $40 \mathrm{cycles}$ of $30 \mathrm{~s}$ at $95^{\circ} \mathrm{C}, 30 \mathrm{~s}$ at $60^{\circ} \mathrm{C}$ and $30 \mathrm{~s}$ at $72^{\circ} \mathrm{C}$. Specificity was verified using melting curve analysis and agarose gel electrophoresis The $C_{\mathrm{t}}$ values of each sample were used in the post-PCR data analysis. Albumin DNA was used as loading controls for all QMSP normalization.

In vivo SAHH activity assay. The experiments were performed in a 96-well scale using the Mouse Homocysteine (Hcy) ELISA Kit (Mybiosource, MBS260152) that allows quantitative measurement of homocysteine concentration in cell extracts, according to the manufacturer's instructions. The concentration of homocysteine in cell extracts was used as a readout for in vivo SAHH activity ${ }^{37}$, as SAHH hydrolyses SAH to homocysteine and adenosine. Briefly, myotubes were washed with cold PBS and lysed on plate in $200 \mu$ lof lysis buffer $(40 \mathrm{mM}$ hexadecyltrimethylammonium bromide, $75 \mathrm{mM}$ Tris- $\mathrm{HCl}, \mathrm{pH} 8.0,1 \mathrm{M} \mathrm{NaCl}$, $15 \mathrm{mM}$ EDTA). The lysate was cleared of insoluble materials by centrifugation at $15,000 \mathrm{~g}$ at $4^{\circ} \mathrm{C}$ for $15 \mathrm{~min}$. Immediately following the centrifugation, $100 \mu \mathrm{l}$ of the supernatant was collected and used for SAHH activity measurement. The absorbance of the samples was determined using a FilterMax F3\&F5 Multi-Mode Microplate Reader (Molecular Devices). A serial dilution of mouse Hcy was included in each assay to obtain a standard curve. The assay was performed in triplicate. The concentrations of DNA and RNA extracted from parallel wells were used as loading controls for SAHH activity normalization.

ChIP. These experiments were performed in a six-well plate scale using the Pierce Agarose ChIP Kit (Thermo Scientific, 26156) according to the manufacturer's instructions with minor modifications. Briefly, myotubes were detached from plates in $0.25 \%$ Trypsin-EDTA and transferred to a $1.5-\mathrm{ml}$ tube. Crosslinking was performed in $1 \%$ formaldehyde at room temperature for $10 \mathrm{~min}$, and the reaction was stopped by glycine. ChIPs using anti-Ser-5(P)-RNAP or anti-DNMT3B antibodies (or rabbit pre-immune IgGs as a negative control) were carried out overnight at $4{ }^{\circ} \mathrm{C}$. Levels of ChIP-purified DNA were determined with RT-qPCR (see Supplementary Table 3 for primer sequences). The relative enrichments of the indicated DNA regions were calculated using the Percent Input Method according to the manufacturer's instructions.

Production of RNA fragments for in vitro analyses. Zymo Taq DNA polymerase (Zymo Research, E2001) was used to amplify hURE (128 nucleotide (nt)), mURE $(122 \mathrm{nt})$ and hNSE $(131 \mathrm{nt})$ using plasmid DNA containing human ${ }^{29}$ or mouse (accession no. NR_001592) H19 sequences as PCR templates. All forward primers contained a T7 promoter sequence (Supplementary Table 4). The PCR products were purified using Zymo DNA clean and concentrator kit (Zymo Research, D4003) and used as templates for subsequent in vitro transcription. RNA fragments for EMSA and in vitro rSAHH activity analysis were generated by in vitro transcription using the T7 MEGAscript Kit (Ambion, AM1334). The resulting RNA was lithium chloride precipitated and the quality of the RNA was confirmed using electrophoresis. The RNA $3^{\prime}$ End Biotinylation Kit (Thermo Scientific, 20160) was used to make $3^{\prime}$-end biotin-labelled RNA fragments, according to the manufacturer's instructions. The biotin-labelled RNA fragments 
were extracted with phenol:chloroform and precipitated with $70 \%$ ethanol. Purified RNA fragments were aliquoted and stored at $-80^{\circ} \mathrm{C}$ until use.

EMSA. Binding reactions were carried out in $20 \mu \mathrm{l}$ containing $5 \mathrm{nM}$ of $3^{\prime}$-end biotin-labelled RNA, together with indicated amounts of Escherichia coli-expressed, purified rSAHH, (Creative BioMart, AHCY-31342TH). Binding buffer contained $5 \mathrm{mM}$ Tris- $\mathrm{HCl}$, pH 7.4, $5 \mathrm{mM} \mathrm{MgCl}_{2}, 3 \%$ (v/v) glycerol, $100 \mathrm{mM} \mathrm{NaCl}, 1 \mathrm{mM}$ DTT, $1 \times$ proteinase inhibitor cocktail (CalBiochem, 539137) and $1 \times$ RNase inhibitor (Roche, 10412600)). Before mixing with the rest of the components in the binding reaction, RNA was heated at $90^{\circ} \mathrm{C}$ for $3 \mathrm{~min}$, followed by immediate chill on ice until use. Binding reaction was conducted at $25^{\circ} \mathrm{C}$ for $30 \mathrm{~min}$. At the end of incubation, $5 \mu \mathrm{l}$ of loading buffer was added and samples loaded on $4 \%$ native polyacrylamide gels (Bio-Rad mini protein gel size), which had been pre-electrophoresed at $4{ }^{\circ} \mathrm{C}$ for $60 \mathrm{~min}$ in $0.5 \times$ Tris-borate-EDTA. Electrophoresis was carried out at $4^{\circ} \mathrm{C}$ at $140 \mathrm{~V}$ for $75 \mathrm{~min}$. Following electrophoresis, components in the gels were transferred to Amersham Hybond- $\mathrm{N}+$ nylon membrane (GE Healthcare, RPN303B) in $0.5 \times$ Tris-borate-EDTA at $400 \mathrm{~mA}$ for $38 \mathrm{~min}$ at $4{ }^{\circ} \mathrm{C}$. The membrane was then crosslinked with ultraviolet light $(254 \mathrm{~nm})$ for $4 \mathrm{~min}$ at a distance of $0.5 \mathrm{~cm}$. Signals were developed using LightShift chemiluminescent EMSA kit (Thermo Scientific, 89880) according to the manufacturer's instructions.

For EMSA competition assays, rSAHH protein and labelled hURE RNA were added to a $20-\mu \mathrm{l}$ reaction at final concentrations of $2 \mu \mathrm{M}$ and $5 \mathrm{nM}$, respectively. Unlabelled hURE, mURE, hNSE and poly(U) (Sigma-Aldrich, P9528-10MG) were added at the indicated molar excess, followed by incubation and electrophoresis, as described above.

Band intensities of scanned films were quantified using the ImageJ software, and the data processed using the GraphPad Prism programme (Version 6.0f). The per cent bound RNA was determined as follows: [\% bound complex $=$ (bound probe - background)/(total probe - background $) \times 100$ ]. All experiments contained a control reaction lacking rSAHH. $K_{\mathrm{d}}$ was calculated using nonlinear regression analysis performed with the Prism 6.0f software. $K_{\mathrm{d}}$ is the dissociation constant. Signals of complexes 1 and 2 were combined to generate probe-bound signals in $K_{\mathrm{d}}$ calculation.

In vitro rSAHH activity assays. These experiments were designed to test whether binding of H19 RNA fragments to rSAHH would inhibit rSAHH enzymatic activity in vitro. The experiments were performed using the Adenosylhomocysteinase Activity Fluorometric Assay Kit (Biovision, K807-100) according to the manufacturer's instructions. This kit allows quantitative detection of adenosine concentration as a readout for rSAHH's ability to hydrolyse SAH in vitro. Briefly, $2 \mathrm{mg}$ of rSAHH was pre-incubated with unlabelled RNA fragments (preheated at $90^{\circ} \mathrm{C}$ for $3 \mathrm{~min}$ and then chilled on ice before use) in AHCY assay buffer in a total volume of $20 \mu \mathrm{l}$ at room temperature for $20 \mathrm{~min}$. At the end of incubation, an additional $30 \mathrm{ml}$ of AHCY assay buffer was added, followed by addition of $50 \mu \mathrm{l}$ of reagents containing SAH. The resulting $100 \mu \mathrm{l}$ reaction mixture was immediately subjected to absorbance reading using a kinetic mode in a FilterMax F3\&F5 MultiMode Microplate Reader (Molecular Devices). In the final 100- $\mu$ l reaction mixture, the concentration of rSAHH was $0.4 \mathrm{mM}$, and those of the RNA fragments were 0 , 0.2 and $1 \mathrm{nM}$. Relative rSAHH activities are presented with those in the absence of the RNA fragments arbitrarily set as 1 .

Methyl-MiniSeq library construction. Libraries were prepared from 200 to $500 \mathrm{ng}$ of genomic DNA digested with 60 units of Taq $\alpha \mathrm{I}$ and 30 units of MspI (NEB) sequentially and then extracted with Zymo Research DNA Clean \& Concentrator-5 kit (Cat. no. D4003). Fragments were ligated to pre-annealed adapters containing $5^{\prime}$-methyl-cytosine instead of cytosine according to the Illumina's specified guidelines (www.illumina.com). Adaptor-ligated fragments of $150-250$ and $250-350 \mathrm{bp}$ in size were recovered from a $2.5 \%$ NuSieve $1: 1$ agarose gel (Zymoclean Gel DNA Recovery Kit, Zymo Research Cat. no. D4001). The fragments were then bisulfite-treated using the EZ DNA Methylation-Lightning Kit (Zymo Research, Cat. no.D5020). Preparative-scale PCR was performed and the resulting products were purified (DNA Clean \& Concentrator, Zymo Research, Cat. no.D4005) for sequencing on an Illumina HiSeq.

Methyl-MiniSeq Sequence alignments and data analysis. Sequence reads from bisulfite-treated EpiQuest libraries were identified using standard Illumina base-calling software and then analysed using a Zymo Research proprietary analysis pipeline, which is written in Python and used Bismark (http://www.bioinformatics.babraham.ac.uk/projects/bismark/) to perform the alignment. Index files were constructed using the bismark_genome_preparation command and the entire reference genome. The non_directional parameter was applied while running Bismark. All other parameters were set to default. Filled-in nucleotides were trimmed off when doing methylation calling. The methylation level of each sampled cytosine was estimated as the number of reads reporting a $\mathrm{C}$, divided by the total number of reads reporting a $\mathrm{C}$ or T. Fisher's exact test or $t$-test was performed for each $\mathrm{CpG}$ site, which has at least five reads coverage, and promoter, gene body and $\mathrm{CpG}$ island annotations were added for each $\mathrm{CpG}$ included in the comparison.
RNA-seq and data analysis. Mouse $\mathrm{C} 3 \mathrm{H}$ myoblasts were maintained in six-well plates. Myotubes were transfected with siCon or siH19 in triplicates for $40 \mathrm{~h}$ following initiation of differentiation. Cells were harvested for RNA extraction $48 \mathrm{~h}$ post transfection using the Purelink RNA mini kit (Cat. no. 12183018A). RNA-seq libraries were prepared using the Illumina TruSeq Stranded Total RNA LT kit with Ribo-Zero Human/Mouse/Rat, setA (Cat. no. rs-122-2201) according to the sample preparation protocol. Briefly, $1 \mu \mathrm{g}$ total RNA was subjected to Ribo-Zero depletion to remove rRNAs. The remaining RNA was purified, fragmented and primed with random hexamers for cDNA synthesis. After first and second cDNA synthesis, cDNA fragments were adenylated and then ligated to indexing adapters. The cDNA fragments were enriched by PCR, purified and then sequenced on an Illumina NextSeq500 using paired-end chemistry and 76-bp cycles. Sequences are available from the GEO with accession number GSE73014.

Illumina BaseSpace (https://basespace.illumina.com/)-embedding tools were used to analyse the RNA-seq data. TopHat Alignment 1.0.0 app was used to map sequencing reads to mm10 genome. Cufflinks Assembly \& DE 1.0.0 app containing Cufflinks 2.1.1 and Cuffdiff 2.1.1 was applied to assemble mapped transcripts and calculate differential expression of genes and transcripts.

Statistical analysis. All data (unless otherwise indicated) are presented as mean \pm s.d. Statistical analyses were performed using the Statistical Package for the Social Science computer software version 17.0 (IBM SPSS Statistics, Chicago, IL, USA). The Student's $t$-test was used to compare differences between quantitative variables. The Fisher's exact text was used when appropriate, for comparing categorical variables (contingency tables). $P$ values at 0.05 or smaller were considered significant.

\section{References}

1. Jin, B. et al. Linking DNA methyltransferases to epigenetic marks and nucleosome structure genome-wide in human tumor cells. Cell Rep. 2, 1411-1424 (2012).

2. Varier, R. A. \& Timmers, H. T. Histone lysine methylation and demethylation pathways in cancer. Biochim. Biophys. Acta 1815, 75-89 (2011).

3. Varley, K. E. et al. Dynamic DNA methylation across diverse human cell lines and tissues. Genome Res. 23, 555-567 (2013).

4. Brenner, C. et al. Myc represses transcription through recruitment of DNA methyltransferase corepressor. EMBO J. 24, 336-346 (2005).

5. Guttman, M. \& Rinn, J. L. Modular regulatory principles of large non-coding RNAs. Nature 482, 339-346 (2012).

6. Cech, T. R. \& Steitz, J. A. The noncoding RNA revolution-trashing old rules to forge new ones. Cell 157, 77-94 (2014).

7. Di Ruscio, A. et al. DNMT1-interacting RNAs block gene-specific DNA methylation. Nature 503, 371-376 (2013)

8. Tehlivets, O., Malanovic, N., Visram, M., Pavkov-Keller, T. \& Keller, W. S-adenosyl-L-homocysteine hydrolase and methylation disorders: yeast as a model system. Biochim. Biophys. Acta 1832, 204-215 (2013).

9. Miller, M. W. et al. The mouse lethal nonagouti $(\mathrm{a}(\mathrm{x}))$ mutation deletes the S-adenosylhomocysteine hydrolase (Ahcy) gene. EMBO J. 13, 1806-1816 (1994).

10. Leal, J. F. et al. S-adenosylhomocysteine hydrolase downregulation contributes to tumorigenesis. Carcinogenesis 29, 2089-2095 (2008).

11. Baric, I. Inherited disorders in the conversion of methionine to homocysteine. J. Inherit. Metab. Dis. 32, 459-471 (2009).

12. De Clercq, E. John Montgomery's legacy: carbocyclic adenosine analogues as SAH hydrolase inhibitors with broad-spectrum antiviral activity. Nucleosides Nucleotides Nucleic Acids 24, 1395-1415 (2005).

13. Wang, Y. et al. Regulation of s-adenosylhomocysteine hydrolase by lysine acetylation. J. Biol. Chem. 289, 31361-31372 (2014).

14. Gabory, A., Jammes, H. \& Dandolo, L. The H19 locus: role of an imprinted non-coding RNA in growth and development. Bioessays 32, 473-480 (2010).

15. Dey, B. K., Pfeifer, K. \& Dutta, A. The H19 long noncoding RNA gives rise to microRNAs miR-675-3p and miR-675-5p to promote skeletal muscle differentiation and regeneration. Genes Dev. 28, 491-501 (2014).

16. Gao, Y. et al. The H19/let-7 double-negative feedback loop contributes to glucose metabolism in muscle cells. Nucleic Acids Res. 42, 13799-13811 (2014).

17. Ohlsson, R., Hedborg, F., Holmgren, L., Walsh, C. \& Ekstrom, T. J. Overlapping patterns of IGF2 and H19 expression during human development: biallelic IGF2 expression correlates with a lack of H19 expression. Development 120, 361-368 (1994).

18. Gabory, A. et al. H19 acts as a trans regulator of the imprinted gene network controlling growth in mice. Development 136, 3413-3421 (2009).

19. Onyango, P. \& Feinberg, A. P. A nucleolar protein, H19 opposite tumor suppressor (HOTS), is a tumor growth inhibitor encoded by a human imprinted H19 antisense transcript. Proc. Natl Acad. Sci. USA 108, 16759-16764 (2011).

20. Venkatraman, A. et al. Maternal imprinting at the H19-Igf2 locus maintains adult haematopoietic stem cell quiescence. Nature 500, 345-349 (2013). 
21. Ghazal, S. et al. H19 lncRNA alters stromal cell growth via IGF signaling in the endometrium of women with endometriosis. EMBO Mol. Med. 7, 996-1003 (2015).

22. Matouk, I. et al. The increasing complexity of the oncofetal h19 gene locus: functional dissection and therapeutic intervention. Int. J. Mol. Sci. 14, 4298-4316 (2013)

23. Monnier, P. et al. H19 lncRNA controls gene expression of the Imprinted Gene Network by recruiting MBD1. Proc. Natl Acad. Sci. USA 110, 20693-20698 (2014).

24. Keniry, A. et al. The H19 lincRNA is a developmental reservoir of miR-675 that suppresses growth and Igf1r. Nat. Cell Biol. 14, 659-665 (2012).

25. Tsang, W. P. et al. Oncofetal H19-derived miR-675 regulates tumor suppressor RB in human colorectal cancer. Carcinogenesis 31, 350-358 (2010).

26. Wang, G. et al. Zbtb7a suppresses prostate cancer through repression of a Sox9-dependent pathway for cellular senescence bypass and tumor invasion. Nat. Genet. 45, 739-746 (2013).

27. Luo, M. et al. Long non-coding RNA H19 increases bladder cancer metastasis by associating with EZH2 and inhibiting E-cadherin expression. Cancer Lett. 333, 213-221 (2013).

28. Giovarelli, M. et al. H19 long noncoding RNA controls the mRNA decay promoting function of KSRP. Proc. Natl Acad. Sci. USA 111, E5023-E5028 (2014).

29. Kallen, A. N. et al. The imprinted H19 lncRNA antagonizes let-7 microRNAs. Mol. Cell 52, 101-112 (2013).

30. Yan, L. et al. Regulation of tumor cell migration and invasion by the H19/let-7 axis is antagonized by metformin-induced DNA methylation. Oncogene 34, 3076-3084 (2014)

31. Castello, A. et al. Insights into RNA biology from an atlas of mammalian mRNA-binding proteins. Cell 149, 1393-1406 (2012).

32. Nagy, E. \& Rigby, W. F. Glyceraldehyde-3-phosphate dehydrogenase selectively binds AU-rich RNA in the $\mathrm{NAD}(+)$-binding region (Rossmann fold). J. Biol. Chem. 270, 2755-2763 (1995).

33. Pioli, P. A., Hamilton, B. J., Connolly, J. E., Brewer, G. \& Rigby, W. F. Lactate dehydrogenase is an AU-rich element-binding protein that directly interacts with AUF1. J. Biol. Chem. 277, 35738-35745 (2002).

34. Kaffer, C. R., Grinberg, A. \& Pfeifer, K. Regulatory mechanisms at the mouse Igf2/H19 locus. Mol. Cell Biol. 21, 8189-8196 (2001).

35. Eun, B. et al. The Igf $2 / \mathrm{H} 19$ muscle enhancer is an active transcriptional complex. Nucleic Acids Res. 41, 8126-8134 (2013).

36. Eun, B., Sampley, M. L., Good, A. L., Gebert, C. M. \& Pfeifer, K. Promoter cross-talk via a shared enhancer explains paternally biased expression of Nctcl at the Igf2/H19/Nctc1 imprinted locus. Nucleic Acids Res. 41, 817-826 (2013).

37. Fernandez-Sanchez, M. E., Gonatopoulos-Pournatzis, T., Preston, G., Lawlor, M. A. \& Cowling, V. H. S-adenosyl homocysteine hydrolase is required for Myc-induced mRNA cap methylation, protein synthesis, and cell proliferation. Mol. Cell Biol. 29, 6182-6191 (2009).

38. Barres, R. et al. Non-CpG methylation of the PGC-1alpha promoter through DNMT3B controls mitochondrial density. Cell Metab. 10, 189-198 (2009).

39. Yang, X. et al. Gene body methylation can alter gene expression and is a therapeutic target in cancer. Cancer Cell 26, 577-590 (2014).

40. Maunakea, A. K. et al. Conserved role of intragenic DNA methylation in regulating alternative promoters. Nature 466, 253-257 (2010).

41. Huang, Y. et al. Inhibition of S-adenosylhomocysteine hydrolase by acyclic sugar adenosine analogue D-eritadenine. Crystal structure of S-adenosylhomocysteine hydrolase complexed with D-eritadenine. J. Biol. Chem. 277, 7477-7482 (2002).

42. Ctrnacta, V. et al. Efficacy of S-adenosylhomocysteine hydrolase inhibitors, D-eritadenine and (S)-DHPA, against the growth of Cryptosporidium parvum in vitro. Exp. Parasitol. 126, 113-116 (2010).
43. Whittle, A. J. et al. BMP8B increases brown adipose tissue thermogenesis through both central and peripheral actions. Cell 149, 871-885 (2012).

44. Shalini, S., Dorstyn, L., Dawar, S. \& Kumar, S. Old, new and emerging functions of caspases. Cell Death Differ. 22, 526-539 (2014).

45. Klingseisen, A. \& Jackson, A. P. Mechanisms and pathways of growth failure in primordial dwarfism. Genes Dev. 25, 2011-2024 (2011)

46. Wang, H., Gauthier, B. R., Hagenfeldt-Johansson, K. A., Iezzi, M. \& Wollheim, C. B. Foxa2 (HNF3beta ) controls multiple genes implicated in metabolismsecretion coupling of glucose-induced insulin release. J. Biol. Chem. 277, 17564-17570 (2002).

47. Sun, X. J. et al. Identification and characterization of a novel human histone $\mathrm{H} 3$ lysine 36-specific methyltransferase. J. Biol. Chem. 280, 35261-35271 (2005).

48. Faber, P. W. et al. Huntingtin interacts with a family of WW domain proteins. Hum. Mol. Genet. 7, 1463-1474 (1998).

49. Kinde, B., Gabel, H. W., Gilbert, C. S., Griffith, E. C. \& Greenberg, M. E. Reading the unique DNA methylation landscape of the brain: non-CpG methylation, hydroxymethylation, and MeCP2. Proc. Natl Acad. Sci. USA 112 6800-6806 (2015).

50. Santiago, M., Antunes, C., Guedes, M., Sousa, N. \& Marques, C. J. TET enzymes and DNA hydroxymethylation in neural development and function-how critical are they? Genomics 104, 334-340 (2014).

51. Wen, L. \& Tang, F. Genomic distribution and possible functions of DNA hydroxymethylation in the brain. Genomics 104, 341-346 (2014).

\section{Acknowledgements}

This work was supported by grant 1-15-BS-084 from the American Diabetes Association to Y.H. and grant R01 HD072418 from the NIGMS to G.G.C.

\section{Author contributions}

Y.H. conceived and orchestrated the project, analysed the data and wrote the manuscript J.Z., L.Y., T.Z., M.M., Y.M. and J.X. carried out the experiments and analysed the data. N.Z. did RNA-seq and data analysis. K.G., H.C. and Z.S. performed genome-wide methylation profiling experiments and data analysis. L.L. and T.Z. designed primers for QMSP. G.G.C. and H.S.T. provided intellectual insights into epigenetic regulation and critical reading of the manuscript.

\section{Additional information}

Accession codes: RNA-seq and methylation profile data are available at the GEO under accession code: GSE73014.

Supplementary Information accompanies this paper at http://www.nature.com/ naturecommunications

Competing financial interests: The authors declare no competing financial interests.

Reprints and permission information is available online at http://npg.nature.com/ reprintsandpermissions/

How to cite this article: Zhou, J. et al. H19 lncRNA alters DNA methylation genomewide by regulating S-adenosylhomocysteine hydrolase. Nat. Commun. 6:10221 doi: $10.1038 /$ ncomms10221 (2015)

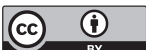

This work is licensed under a Creative Commons Attribution 4.0 International License. The images or other third party material in this article are included in the article's Creative Commons license, unless indicated otherwise in the credit line; if the material is not included under the Creative Commons license, users will need to obtain permission from the license holder to reproduce the material. To view a copy of this license, visit http://creativecommons.org/licenses/by/4.0/ 